\title{
Phase synchronization and criticality in a network of neural mass models
}

\section{Sheida Kazemi}

Tarbiat Modares University

Yousef Jamali ( $\square$ Y.Jamali@modares.ac.ir)

Tarbiat Modares University

\section{Research Article}

Keywords: neural mass models, synchronization, memory, learning, perception

Posted Date: April 20th, 2021

DOI: https://doi.org/10.21203/rs.3.rs-437925/v1

License: (c) (i) This work is licensed under a Creative Commons Attribution 4.0 International License. Read Full License 


\title{
Phase synchronization and criticality in a network of neural mass models
}

\author{
Sheida Kazemi ${ }^{1}$ and Yousef Jamali ${ }^{1, *}$
}

\author{
${ }^{1}$ Biomathematics Laboratory, Department of Applied Mathematics, School of Mathematical Sciences, Tarbiat \\ Modares University, Tehran, Iran \\ *Y.Jamali@modares.ac.ir
}

\begin{abstract}
Synchronization has an important role in neural networks, and their dynamics are mostly accompanied by cognitive activities such as memory, learning, and perception. These activities arise from the collective neural behaviors and are not totally understood yet. This paper aims to investigate a cortical model from this perspective. In this paper, we investigated a network of neural populations in a way the dynamics of each node corresponded to the Jansen-Rit neural mass model. First, we put this dynamic on a single mass of four different input levels. Then, we considered a Watts-Strogatz network of Jansen-Rit oscillators. We observed an epileptic activity in the weak input level. The network to change various parameters is considered. The detailed results including the mean times series, phase spaces and power spectrum revealed a wide range of different behaviors such as epilepsy, unimpaired, and a transition between synchrony and asynchrony states. Since the critical state is a dynamic candidate for healthy brains, we considered some measures of criticality and investigated them on phase transition points. We showed that the criticality hypothesis is not all or nothing theory. It means that due to the nature of specific indicators selected for studying the criticality, the phase transition point can be a critical point or not. Indeed, some markers of criticality can exist in phase transition points, and others may not. As a result, we do not claim that neural models show criticality or not, but we can only admit that they have a percentage of criticality.
\end{abstract}

\section{Introduction}

Synchronization is a process that the elements of the system, coordinate one dynamical property by coupling or external force. The simplest of synchronization is full synchronization proposed by Fujisaka and Yamada in 1983 ${ }^{1}$. In 1996, Rosenblum was the first one who introduced the concept of phase synchronization ${ }^{2}$. Due to this definition, the phase difference will be fixed (smaller than $2 \pi$ ), but amplitudes not necessarily the same in this definition. At least two elements exist in the synchronization process. To investigate network synchrony, we first assume that $\mathrm{N}$ oscillators interact with each other. Each oscillator state shows a fixed-point attractor (steady-state), a limit cycle (a closed cycle that shows periodic oscillations), or chaos (a complex orbit that determines aperiodic oscillations). Measurement of synchronization in neural signals is a procedure to estimate oscillatory activities $^{3-5}$. The rhythmic neural interaction can measure by different linear (e.g., cross-correlation) or nonlinear (e.g., mutual information) methods that analyze signals in time and frequency spaces ${ }^{6}$.

Synchronization measures have been wildly interested in investigating neural dynamics ${ }^{7-10}$. Especially, synchronization phenomena have a substantial role in determining normal ${ }^{11,12}$ and abnormal ${ }^{13}$ brain function and also in a deeper understanding of information processing ${ }^{14-16}$. Moreover, synchronization influences on prediction and detection of some disorders such as epilepsy $^{17}$, Alzheimer ${ }^{18}$, autism ${ }^{19}$, and schizophrenia ${ }^{20}$.

About $1 \%$ of people suffer from epilepsy ${ }^{21}$. Many patients can control their disease by taking medicine, but approximately $30 \%$ of them have drug-resistant epilepsy. The dynamics of epilepsy disorder is very complex, and it arises from high synchrony of neuron activities ${ }^{22}$. The significant changes in the brain dynamics during epilepsy can be fatal, and in certain conditions, lead to loss of consciousness, body tremors, and even death. To enhance knowledge of the mechanism of epilepsy is imperative.

The biophysical modeling of the brain is an active area for neuroscientists. In this approach, they simulate a complex system by mathematical and physical tools to predict the influence of different biological factors ${ }^{23-25}$. This attitude causes a profitable framework in neural dynamics modeling with large-scale approaches ${ }^{26-29}$. Some cognitive activities such as memory, learning, and perception arise from a mass activity of neurons. There are different scales to study the brain function between a small patch of cortex and a large-scale system. Coupling a collection of neural mass models into mesoscale circuits can bridge between these scales. The population of neurons coupled together to form a neural mass and they can model different phenomena and generate a variety of their dynamics behaviors.

Neural mass models have a long history. Lopez da silva ${ }^{30,31}$, Jansen and Rit ${ }^{32,33}$, Wendling ${ }^{34,35}$, Wilson-Cowan ${ }^{36-39}$, Freeman $^{40-42}$, and Wong-Wang ${ }^{43,44}$ are some models that investigated the collective behavior of neurons. These models are 
used to study different rhythms, the source of them, and also the transition between normal activity in the brain and epilepsy. Moreover, the complexity of electroencephalogram (EEG) shows the complexity of cortical columns that can transform into a simple circle by using neural models, and accordingly, analysis of them is conceivable. The comparison of neural mass models has received a lot of attention. $\mathrm{In}^{45}$, the dynamics of Freeman and Wilson-Cowan models had been analyzed simultaneously. Also, in ${ }^{46}$, two neural mass models have been comprised by different measures. Because synchronization is an appropriate measurement for neural dynamics, it has been shown as an ordered parameter in many research ${ }^{38,45,47-50}$. In brain networks, the coupling strength between units ${ }^{48,49,51}$ and the amount of external input of nodes ${ }^{33,52,53}$ are two interesting parameters to analyze. Both of them have a significant role in developing a wide range of activities. ${ }^{54-56}$. Indeed, neural mass models can show different behaviors due to these parameters and initial conditions that vary in each run. The purpose of this paper is to analyze the dynamics of the Jansen-Rit model that arose to extend simultaneous electrical activities simulation, especially alpha rhythm, in neural masses. Also, Jansen and Rit demonstrated that their model simulates evoked potential. The disability of making different rhythms, especially onset activity of epilepsy, is the main disadvantage of this model.

Since 1988, the Jansen-Rit model has been discussed analytically and numerically ${ }^{54,57-60}{ }^{41}$ clarifies the relationship between structural and functional matrix in a network with this dynamic. Moreover, the concept of criticality was studied in a single column with Jansen-Rit dynamics ${ }^{61}$.

Many research such as ${ }^{41,55,62}$, used empirically measured individual structural connectivity. Structural links of cortical neurons in a network show a small-world topology ${ }^{63}$, so several studies surveyed small-world networks of interconnected neural mass models ${ }^{38,64-66}$.

In this paper, we model the brain as a system of coupled oscillators in the mesoscale approach. First, we explain the Jansen-Rit model in a single unit and then in a network and alter the external input in four levels. We propose cross-correlation measures for synchronization in the materials and methods section. This network shows a phase transition between physiological and epilepsy-like behavior in the weak external input level. We considered the coupling coefficient between nodes and synchronization as control and order parameters, respectively. Next, the synchronization diagram according to the coupling coefficient with synchrony measures has been drawn. Finally, to recognize critical behavior, some markers of its such as high fluctuations (weak stability) and being on the edge of synchronization, neural avalanches with power-law distribution of size, and LRTC (long-range temporal correlation) in the amplitude of neural oscillations has been selected. Our results show that each phase transition point can be regarded as critical, and just a phase transition point does not necessarily show criticality due to other markers.

\section{Materials and Methods}

\section{Graph Theory}

Modeling the brain as a complex network is a powerful mathematical tool to understand the structural and functional of the brain architecture.

Structural brain networks can be shown as graphs that their nodes (vertices) are related to neural elements (neurons or brain regions) and linked by physical connections (edges). Using adjacency matrix $(M)$ is a simple method to display a matrix where $M_{i j}=1$, if there is a connection between node $\mathrm{i}$ and node $\mathrm{j}$ and $M i j=0$ otherwise. In this work, neural populations are considered as nodes, and edges are interpopulation connections.

The clustering coefficient computes how connected a node's neighbors join together to make a cluster. This feature in a network is defined as the average clustering coefficient of nodes. The shortest path length is the least number of edges between nodes and the average of this property shows the average node to node distance in a graph. This concept represents how rapidly information can be transformed through the network.

Regular, random, and small-world networks are three important network models which have largely been studied so far. In regular networks, each node has exactly the same number of connections and the clustering coefficient is high in these networks. The connections between each node in random topology follow a normal degree distribution with a low level of clustering. The average path length is short (long) in regular (random) systems.

Regular, random, and small-world networks are three important network models which have largely been studied so far. In regular networks, each node has exactly the same number of connections and the clustering coefficient is high in these networks. The connections between each node in random topology follow a normal degree distribution with a low level of clustering. The average path length is short (long) in regular (random) systems. Evidence suggested that the real-world networks have small-world properties including two independent structural features, namely, short average path length (long-range connections) and high clustering coefficient. The Watts-Strogatz (WS) models were generated as the simplest networks that have the small-world properties. 


\section{Jansen-Rit model}

In this paper, neural masses are defined as columns. The model consists of a main population of pyramidal neurons that receive inputs from three resources: excitatory and inhibitory interneurons feedback in the same column and external input from other columns. Figure 1 is a schematic representation of this model. We consider a network with an interactive population,

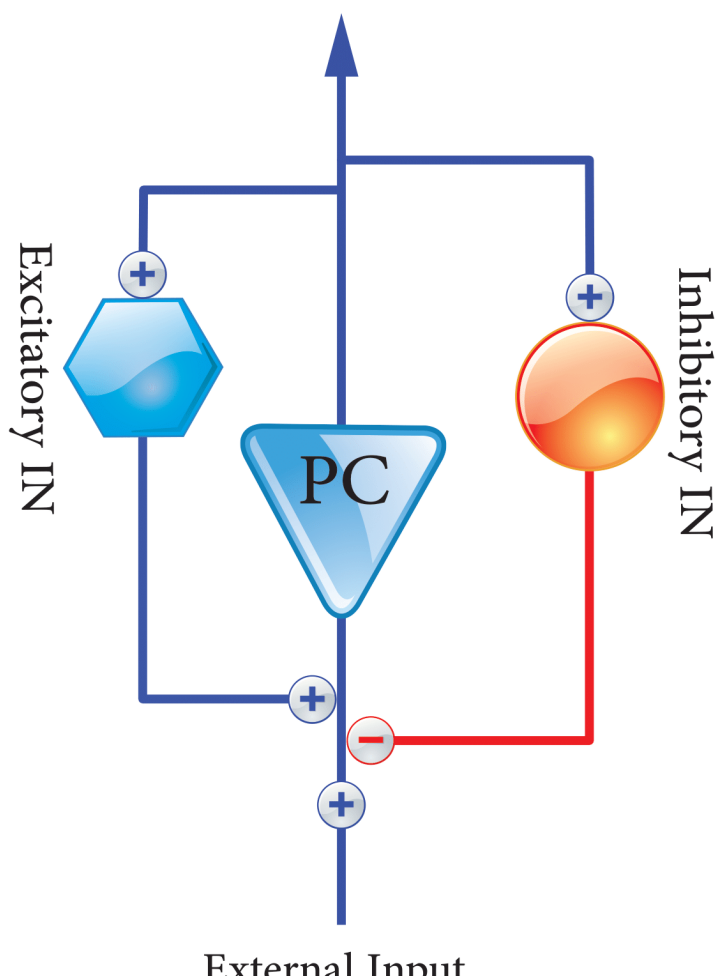

Figure 1. Schematic representation of Jansen-Rit model. A main population (pyramidal cell) interacts with two populations: excitatory interneuron (left) and inhibitory interneuron (right).

representing a patch of the cortex. Each node indicates a neural mass with Jansen-Rit dynamics. Nodes can interact with each other and generate different behaviors. First, we represent this dynamic per node $k, k=1, \ldots, N$. The equations are written by three second-order differential equations and can rewrite with six first-order differential equations as follow ${ }^{54}$ :

$$
\begin{aligned}
& \dot{y}_{0}(t)=y_{3}(t) \\
& \dot{y}_{3}(t)=A a S\left(y_{1}(t)-y_{2}(t)\right)-2 a y_{3}(t)-a^{2} y_{0}(t) \\
& \dot{y}_{1}(t)=y_{4}(t) \\
& \dot{y}_{4}(t)=A a\left\{p(t)+J_{2} S\left[J_{1} y_{0}(t)\right]\right\}-2 a y_{4}(t)-a^{2} y_{1}(t) \\
& \dot{y}_{2}(t)=y_{5}(t) \\
& \dot{y}_{5}(t)=B b J_{4} S\left(J_{3} y_{0}\right)-2 b y_{5}(t)-b^{2} y_{2}(t)
\end{aligned}
$$

Where $(y 0, y 3),(y 1, y 4)$ and $(y 2, y 5)$ are activities of pyramidal, excitatory and inhibitory ensembles respectively. $\mathrm{S}$ is a sigmoid function, transforms the average membrane potential of neurons to mean firing rate of action potentials, and identified as follows:

$$
S(v)=\frac{v_{\max }}{1+e^{r\left(v_{0}-v\right)}}
$$

P means an external input, which can be considered as a noise or input from other columns. Generally, it's chosen from a uniform distribution ranging from 120 and 320 .

Other parameters are quantified in Table $1^{42-44}$. The output of this model is the membrane potential of pyramidal neurons 
Table 1. Parameters in the Jansen-Rit model are obtained experimentally.

\begin{tabular}{|c|c|c|}
\hline Parameters & Interpretation & Value \\
\hline$A$ & Amplitude of excitatory PSP & $3.25 \mathrm{mV}$ \\
\hline$B$ & Amplitude of inhibitory PSP & $22 \mathrm{mV}$ \\
\hline $1 / a$ & Time constant of excitatory PSP & $a=100 s^{-1}$ \\
\hline $1 / b$ & Time constant of inhibitory PSP & $b=50 s^{-1}$ \\
\hline$J 1, J 2$ & Average numbers of synapses between excitatory neural populations & $1 * J, 0.8 * J$ \\
\hline$J 3, J 4$ & Average numbers of synapses between inhibitory neural populations & $0.25 * J$ \\
\hline$J$ & Average numbers of synapses between neural populations & 135 \\
\hline$v_{\max }$ & Maximum firing rate & $5 \mathrm{~Hz}$ \\
\hline$v 0$ & Potential at half of maximum firing rate & $6 \mathrm{mV}$ \\
\hline$r$ & Slope of sigmoid function at $v 0$ & $0.56 \mathrm{mV}^{-1}$ \\
\hline
\end{tabular}

$(y 1-y 2)$. Also $J_{i}=J \alpha_{i}$, for $\mathrm{i}=1, \ldots, 4$. Now, the Jansen-Rit's dynamical equations in a network with $\mathrm{N}$ nodes, $\mathrm{i}=1, \ldots, \mathrm{N}$, are as follows ${ }^{41}$ :

$$
\begin{aligned}
& \dot{y}_{0_{i}}(t)=y_{3_{i}}(t) \\
& \dot{y}_{3_{i}}(t)=A a S\left(y_{1_{i}}(t)-y_{2_{i}}(t)\right)-2 a y_{3_{i}}(t)-a^{2} y_{0_{i}}(t) \\
& \dot{y}_{1_{i}}(t)=y_{4_{i}}(t) \\
& \dot{y}_{4_{i}}(t)=A a\left\{p_{i}(t)+\alpha \sum_{j=1}^{N} M_{i j} S\left(y_{1_{j}}(t)-y_{2_{j}}(t)\right)+J_{2} S\left[J_{1} y_{0_{i}}(t)\right]\right\}-2 a y_{4_{i}}(t)-a^{2} y_{1_{i}}(t) \\
& \dot{y}_{2_{i}}(t)=y_{5_{i}}(t) \\
& \dot{y}_{5_{i}}(t)=B_{b} S\left(J_{3} y_{i} 0\right)-2 b y_{5_{i}}(t)-b^{2} y_{2_{i}}(t)
\end{aligned}
$$

Where $M_{i j}$ shows the adjacency matrix of network and $\alpha$ represents coupling coefficients between nodes.

\section{Pearson cross-correlation}

Neural interactions can be measured by multiple criteria, each with its advantages and disadvantages ${ }^{45}$. The simplest measure of non-directed interactions between random variables is Pearson cross-correlation (Pcc) which measures the linear relationship between each pair of random variables. This coefficient removes the temporal structure, so time series are considered as generalizations of random variables. Normalized Pcc coefficient in one-dimensional between two time-series $x$ and $y$ is defined as follow:

$$
r=\frac{\sum_{i=1}^{N}\left(x_{i}-\langle x\rangle\right)\left(y_{i}-\langle y\rangle\right)}{\sqrt{\sum_{i=1}^{N}\left(x_{i}-\langle x\rangle\right)^{2}} \sqrt{\sum_{i=1}^{N}\left(y_{i}-\langle y\rangle\right)^{2}}}
$$

Where $\mathrm{N}$ shows the length of the signal and $\langle x\rangle$ is the mean of time series $x$. The range value of this coefficient varies between -1 and 1 . The quantity of $1(-1)$ represents a perfect linear positive (negative) correlation and 0 means no correlation between two series (uncorrelated state). Note that the matrix constructed by this definition is symmetric and elements are one in the main diagonal.

\section{Detrended fluctuation analysis (DFA)}

DFA method specifies the self-affinity of a signal and demonstrates long-range correlation in time series, which was first introduced by Peng et al in $1994^{46}$. Illustration of this procedure consists of the following five steps:

- Step 1: We consider time-series $\mathrm{x}$ with a length of $\mathrm{N}$ and calculate the cumulative sum of $x-\langle x\rangle$ where $\langle x\rangle$ is the mean signal of $x$ :

$$
Y_{t}=\sum_{i=1}^{t}\left(x_{i}-<x>\right)
$$

where $t \in N$.

- Step 2: $Y_{t}$ is divided into $N_{s}=(N / s)$ segments with the same size s. It is possible that $(N / s)$ not to be an integer, so we do this division from the end of signals. Therefore, we have $2 N_{s}$ segments. Then, the least-squares fit is calculated by minimizing the squared errors in each segment. 
- Step 3: In each segment, the root mean square (RMS) for $Y$ is calculated as follow:

$$
F(s)=\sqrt{\frac{1}{2 N_{s}} \sum_{t=1}^{2 N_{s}}\left(Y_{t}-Y_{\text {local }}(t)\right)^{2}}
$$

which $Y_{\text {local }}$ shows the local trend.

- Step 4: We repeat step 3 for different interval sizes ranges between 4 and $N / 10$ to find the relation between the scaling exponent and the data fluctuation.

- Step 5: We plot $F(s)$ consistent with $\mathrm{s}$ in a logarithm scale. In power-law relation, $F(s) \sim s^{a}$ which $a$, fluctuation or scaling exponent, is determined as the slope of a straight line fit. $0.5<a<1$ represents long-range correlation.

We used Nolds package in Python with no overlapping windows to perform this technique (https://pypi.org/project/nolds/).

\section{Simulation}

In this simulation, we considered a small-world network (Watts-Strogatz network with rewire probability equal to 0.2 ) with 80 oscillators as nodes. Each node is connected to 20 neighbors, 10 on each side. We applied Jansen-Rit dynamics in all nodes and used the stochastic Runge-Kutta method to simulate the system ${ }^{67}$. The time step was set to $10^{-4} \mathrm{~s}$. The coupling coefficient between neurons in each mass was considered 135 (to show alpha rhythm in each single node). Each node received external inputs from other brain regions. First, we supposed that the coupling coefficient between nodes was equal to zero and then increased it. External input interval was $[120,320]$ which we divided into four segments. Based on the permitted input interval, we analyzed the dynamics of the network for four different inputs named small, intermediate, strong, and ultra-strong input. For each of them the input was Gaussian random noise with mean of 145 , 195, 245, and 295 respectively and sigma $=3.25$ for all of them. The length of this simulation was 200 seconds and ran ten times on each coupling coefficient. Elimination of the first 5 seconds of simulation, gave us the certainty that the system achieved to its equilibrium.

\section{Results and discussion}

\section{Synchronization phase transition}

First, we applied the Jansen-Rit model on a single node. The power spectrum of the output signal for four levels of input is shown in figure 2. Increasing input increases the dominant frequency, but all of them are still in the alpha band. Then, we

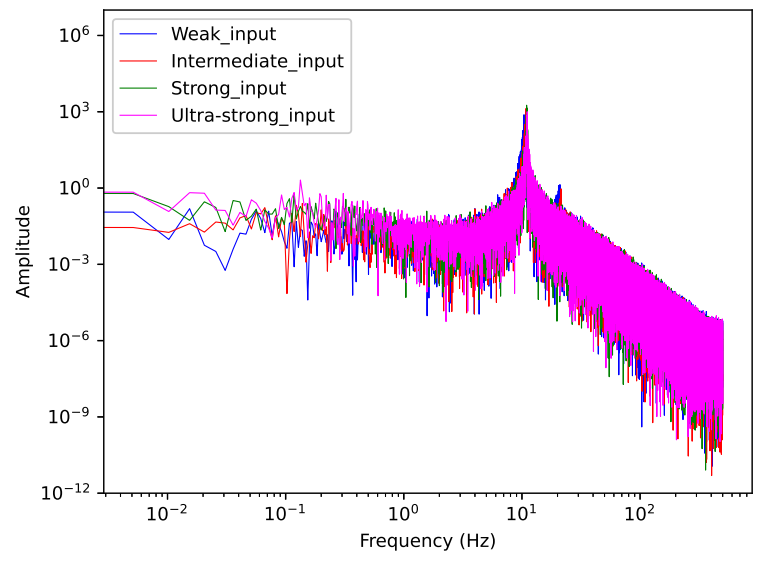

Figure 2. Power spectrum for a single unit in different level inputs. Alpha rhythm is dominant in all of them.

consider a network with an interactive population, representing a patch of the cortex. Each node shows a neural mass with Jansen-Rit dynamics. There are a couple of factors that can influence the network's dynamics. One of the most important of this is coupling strength. Nodes can interact with each other and generate different dynamics behaviors. Changing the coupling coefficient between nodes and analysis of network dynamics has been interested recently ${ }^{35,38,39}$. 
At the start, we assumed that each node received the external input from a weak range. Cross-correlation is a popular method to investigate and compare time series. By considering this time series of each node in the model and calculating Pcc between them, a square matrix was created. The ij'th element of the matrix shows the Pcc of node $\mathrm{i}$ and $\mathrm{j}$ dynamics. The mean of the Pcc matrix for weak input is shown in figure 3. Vertical red bars represent dispersions for ten runs (standard deviations). No

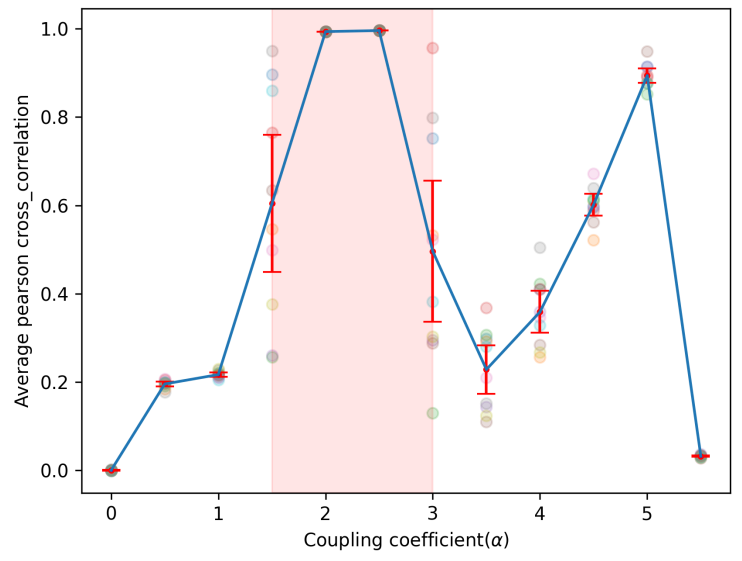

Figure 3. Mean of the Pearson cross-correlation matrix according to the coupling coefficient in a weak input area. In $\alpha=1.5$ and 3, the dispersion of the mean of the Pcc are high and the area between them is related to the epilepsy regime.

coupling leads to zero cross-correlation. It can be seen in figure 3 increasing coupling up to 1.5 causes more correlation. In addition, the mean signal amplitude gets larger (first column in figure 4). During this range of coupling strength, the dominant frequency is still in the alpha band ( $\alpha=0$ in figure 5(A)). At a coupling coefficient equal to 1.5, the network's behavior abruptly has been changed, and as it can be seen in figure $6(\mathbf{C})$, the large amplitude oscillations appear. In each run, switching from high to low amplitude oscillations occurs at different times. In addition, its phase space is exhibited in figure 6(D). It is visible that in this coupling coefficient, the phase space is composed of 2 limit cycles. The first of which in respect of high amplitude oscillations show rhythmic spiking activity, and the second corresponding to a shorter amplitude signal. This transition between high and low amplitude signals is one of the most indicators of brain disorders such as epilepsy ${ }^{68-70}$. High amplitude signals are rhythmic spiking activities represented in theta band frequency and show epileptic activity that the frequencies of recorded epileptic seizure confirm this ${ }^{68}$. As well as, the dominant frequency of the short amplitude regime is alpha. Indeed, this behavior is mixed epileptic-alpha activity. It means that the ictal activity occurs ${ }^{58}$ and it gradually slows down in a non-specific time during the whole simulation due to the stochastic noise and different initial conditions in each run. As a matter of fact, a spontaneous transition happens between two types of behaviors.

Results indicated that in the [1.5,3] regime of coupling strength, the output signal for all repetitions shows only two types of behavior: high amplitude oscillations that either change to low amplitude oscillations or not (figure 6). Notably, the length of this simulation was 200 seconds. Indeed, epileptic activity is seen in this area that can be continued up to 200 seconds or change to a low amplitude oscillatory state. Consequently, we call this area an epileptic regime, and the coupling coefficient equal to 1.5 (3) is the starting (ending) point of epilepsy.

Interestingly, that bistability and transition between periodic areas with high and low amplitudes are the two most features in the investigation of epilepsy ${ }^{69}$.

Fast Fourier Transforms (FFTs) for the values of 1.5, 2, 2.5, and 3 show a significant change in their appearance (figure 5(A)), which confirms a disorder in network function $69,71,72$.

Figure 5(B) shows the dominant frequency according to different coupling coefficients. The color of each cell represents the dominant frequency of an independent in-silico experiment (run). The vertical axis is corresponding to the coupling strength value of each run in its raw. There is a jump phenomenon in frequency in the [1.5-3] regime that is a key feature to realize the epileptic seizure dynamics ${ }^{69,73,74}$. Exclusive of this phenomenon, it is clear that the coupling coefficient and dominant frequency have an inversion relation. Also, presencing bistability in frequency at $\alpha=3$ is an interesting result.

Based on figure 3, a small increase in coupling coefficient more than 1.5 causes a dramatic rise in the mean of the Pcc, and the network has been brought into the maximum value of synchronization. Besides, it can be viewed that there is a big dispersion of the mean of the Pcc matrix in bifurcation points (1.5 and 3). Actually, in the bifurcation points, the network's dynamic changes dramatically. In coupling coefficient equal to 3 , the mean of the Pcc has a great variety, and the variance of 
A

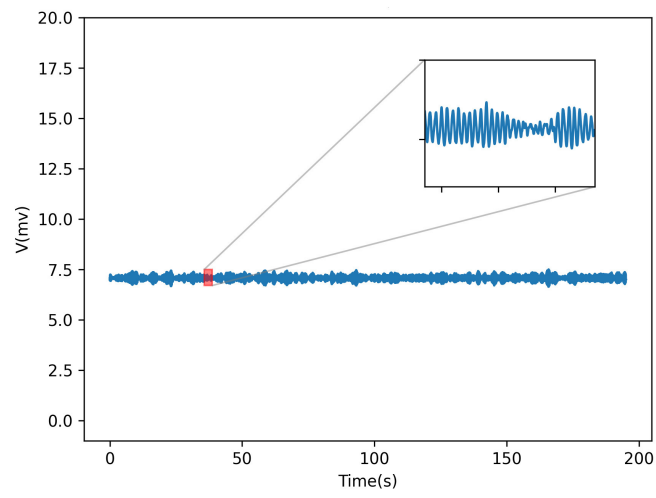

C

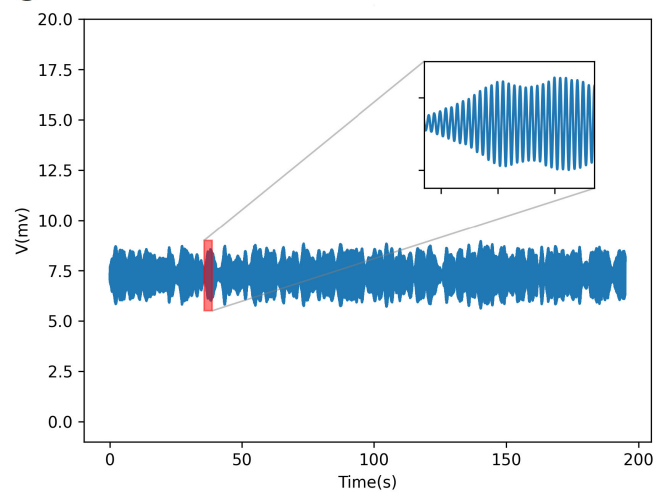

$\mathbf{E}$

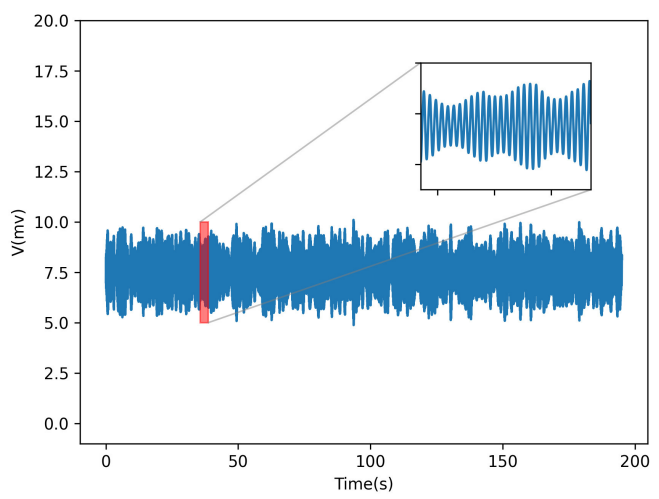

B

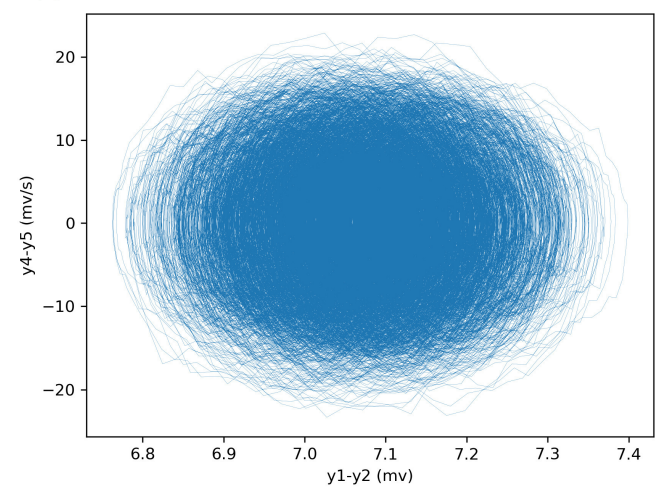

D

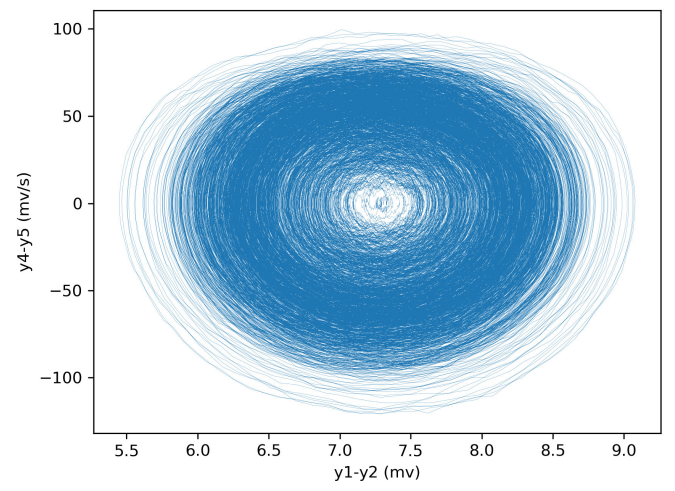

F

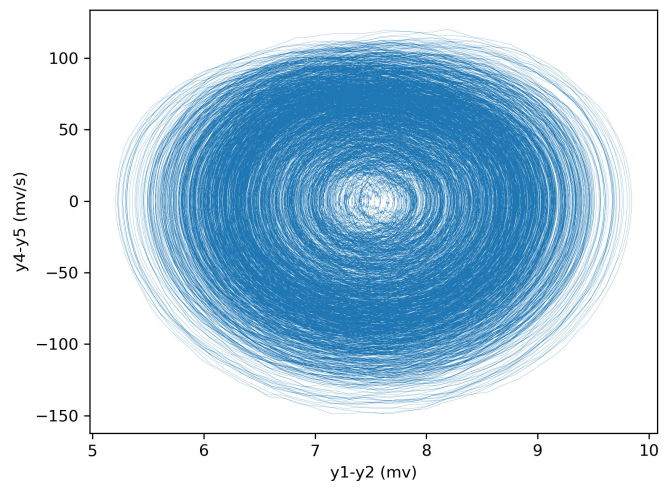

Figure 4. Time series of the mean of the network activity for $\alpha=0(\mathbf{A}), 0.5(\mathbf{C})$, and $1(\mathbf{E})$ and their phase space $(\mathbf{B}, \mathbf{D}, \mathbf{F})$. The $\mathrm{x}$-axis and $\mathrm{y}$-axis show the output signal and its derivation, respectively. By increasing the coupling coefficient, the mean signal amplitude gets larger. The inset of (A), (C) and (E) shows a specific segment of signal for 3 seconds. 
A

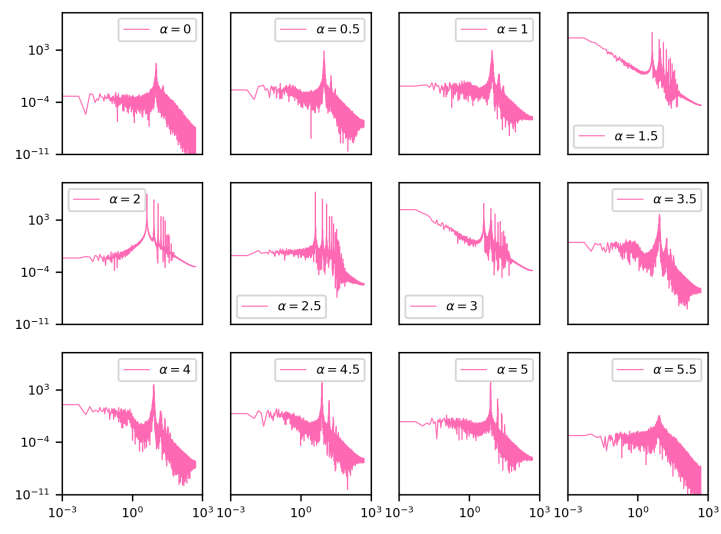

B

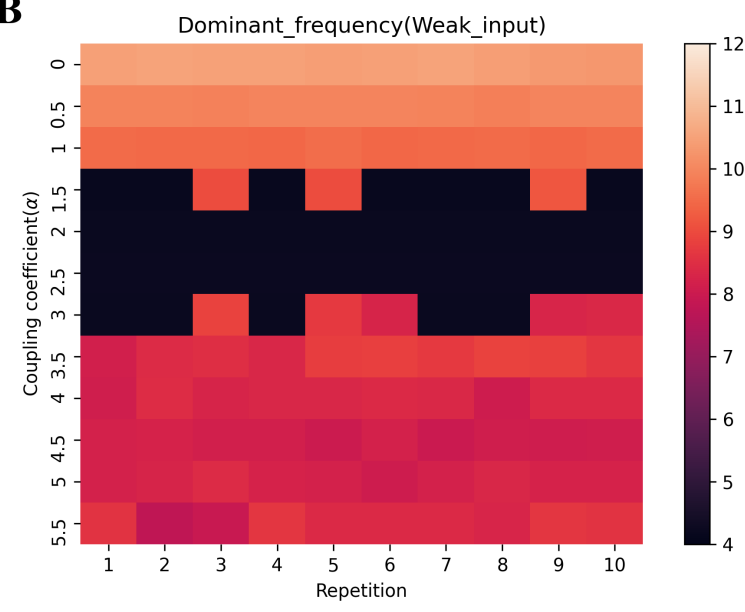

Figure 5. (A): Power spectrum for different coupling coefficients in weak noise. Unusual behavior in $\alpha=1.5,2,2.5$, and 3 is visible. Except in [1.5-3] regime, the dominant frequency is in the alpha band. (B): The value of dominant frequency in different coupling coefficient for ten runs. A jump phenomenon in frequency in the [1.5-3] regime is seen.

them is high. There are more fluctuations of the mean of the Pcc in 3 rather than other coupling coefficients. Cross-correlation matrices in the value of 3 are shown in figure 7. There are different patterns in these matrices. The red (blue) color shows full synchronization (anti-synchronization). Also, in this figure, local and global synchronization can be seen. This issue is determined with red and blue masses, respectively. Local synchronization means that some ensembles of neurons have the same behavior, and some of them act completely differently, i.e., synchronized clusters. Simply put, full red Pearson cross-correlation matrices mean that the network shows a global synchrony state, and the simultaneous presence of blue and red masses in a matrix is a symbol of in-phase and anti-phase local synchronization. This different pattern could be reminiscent of different synchronization patterns of brain networks during different tasks ${ }^{38}$. Again, we followed the rise in coupling strength. It is visible that the decrease in the mean of the Pcc is continued up to 3.5. Figure 8 displays that the mean signal output goes back to the normal state and put in the alpha band frequency $(\alpha=3.5$ in figure 5 (A)).

More increase in $\alpha$ from 3.5 to 5 causes a growth in the mean of the Pcc up to reach maximum value. If we increase $\alpha$ further, this synchrony behavior switches to a fixed-point state, i.e., each node leaves the limit cycle and collapses to a resting state (fixed-point). Mean signals and their phase space for one repeat are shown in figures 8 and 9 , respectively. Moreover, their power spectrum for $\alpha=3.5,4,4.5$, and 5 in figure 5(A) shows a switch from disorder to the normal state, and the dominant frequency of the network goes into an alpha rhythm.

So far, the amount of external input of each node was assumed from a weak level. Here, we consider the input value in the other three ranges (medium, strong, and ultra-strong) and report gathered results.

The plot of the mean of the Pcc matrix for four inputs versus coupling strength has shown in figure 10(A). Moreover, for better understanding, figure 10(B) is a heat-map of the mean of the Pcc matrix for ten independent runs in each of them, where the $\mathrm{x}$-axis is coupling coefficients, and the $\mathrm{y}$-axis is input signals. For all different intensities of input, the correlation between units for the zero couplings is too low. Because in this case, oscillators can not have a significant effect on each other. We expected that the weak input level went forward as specified expected behavior trend in [1-3] in figure 10, however the dynamics of the network due to set parameters seem very complex and unpredictable. It is obvious that lower intensity input needs a higher coupling coefficient to reach its Pcc maximum. Each unit receives some inputs from its neighbors plus the stochastic amount chosen from an interval. Strengthening coupling between nodes leads to receive more from neighbors, and as a result, less stochastic value is needed. Indeed, the input is a fundamental element, and its variations can impress the amount of correlation and the rate of the upward trend to reach the highest degree of this. Interestingly, though plots in each intensity of input have been shifted to the left side, the dispersions of the mean of the Pcc matrix at the $\alpha=1.5,3$ are still high.

According to figure 11, one impressive event is viewed: a jump frequency in the weak level input that is related to the epileptic regime and has been confirmed in ${ }^{69}$. Furthermore, in each input level (except in weak), increasing coupling coefficients result in the reduction of the dominant frequency but remain in the alpha band frequency. 
A

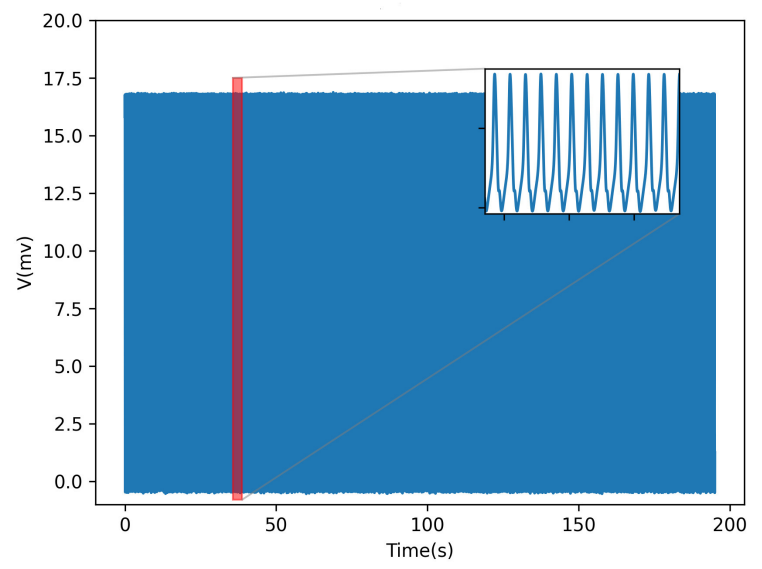

C

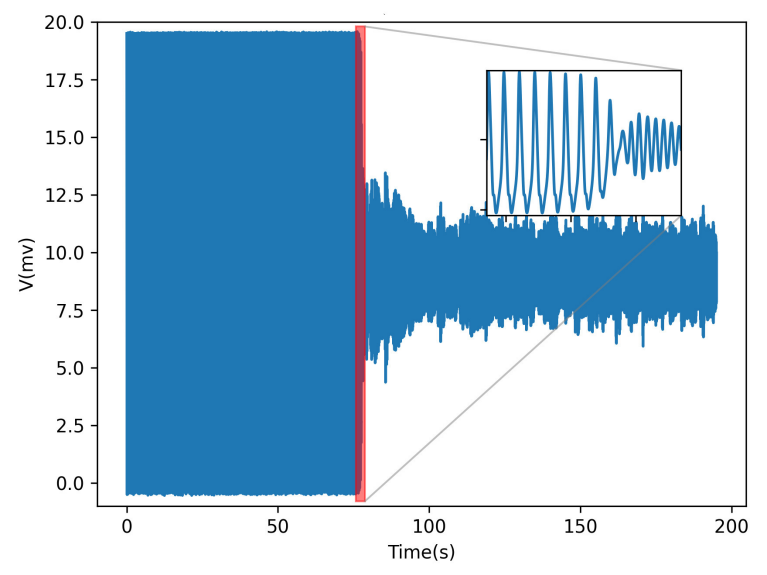

\section{B}

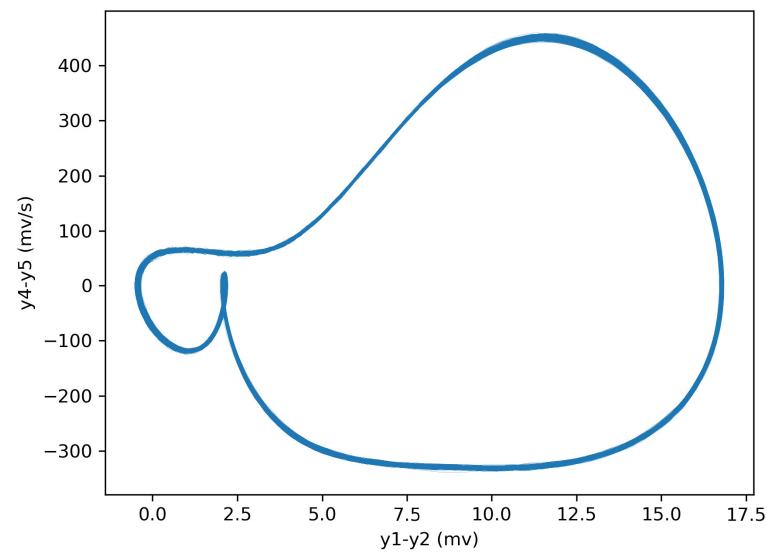

D

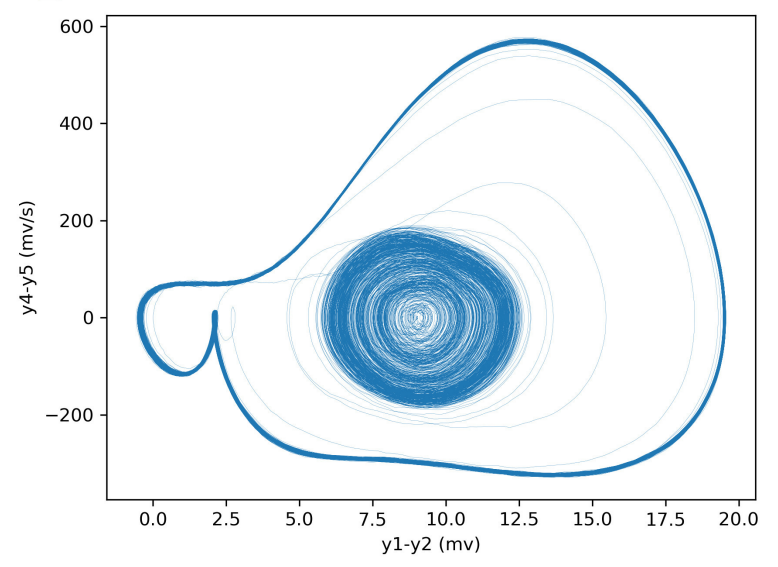

Figure 6. Two possible behaviors in [1.5-3] area ((A), (C)). High amplitude oscillations appear in this regime that either change to low amplitude oscillations (panel (C) or not (panel (A). Their phase spaces is composed of 2 limit cycles (panel (D) or just one limit cycle (panel (B) respectively. The inset of (A) and (C) shows a specific segment of signal for 3 seconds. 

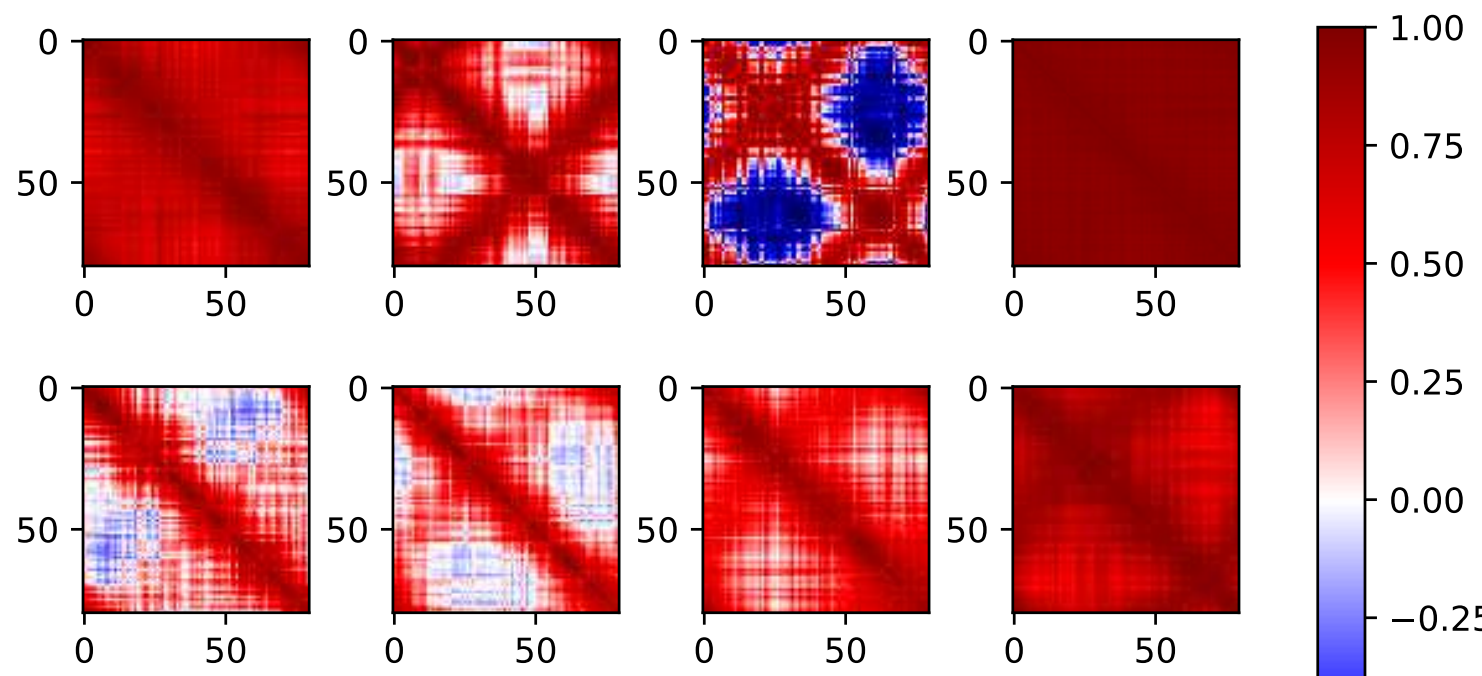

$-0.25$
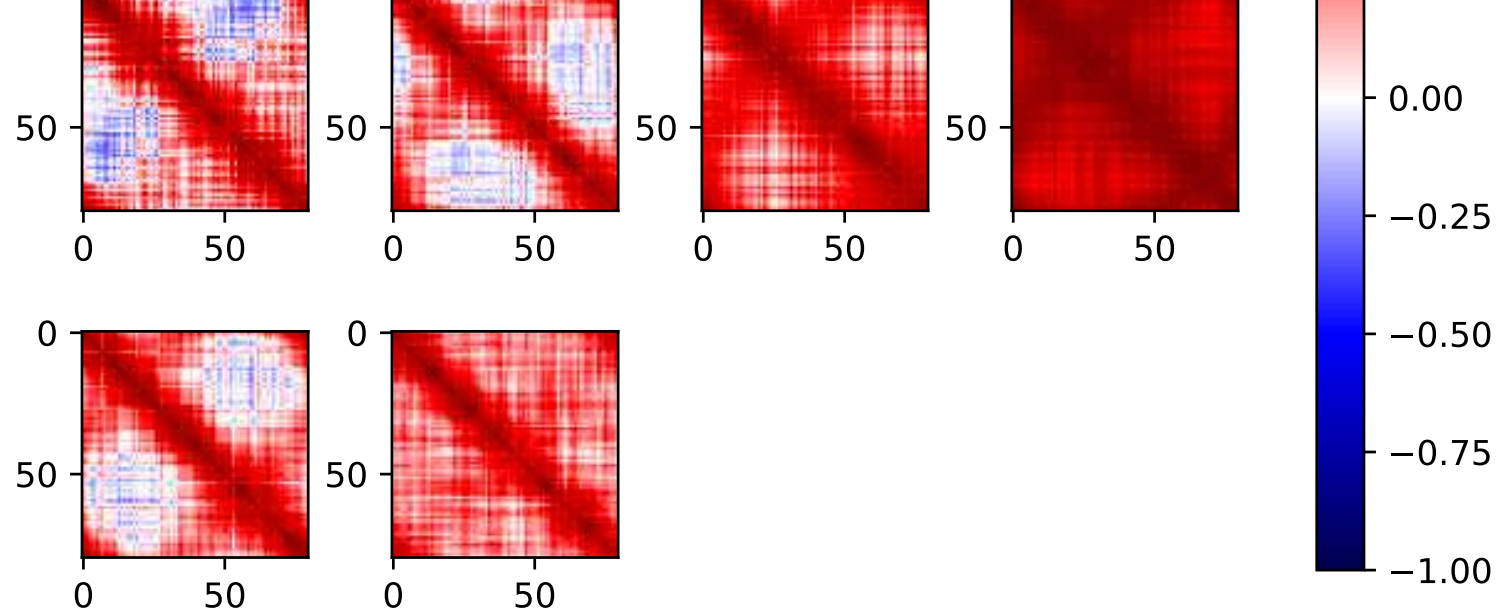

Figure 7. Correlation matrices in $\alpha=3$ over 10 repetitions during weak input. Local and global synchronizations are seen. 
A

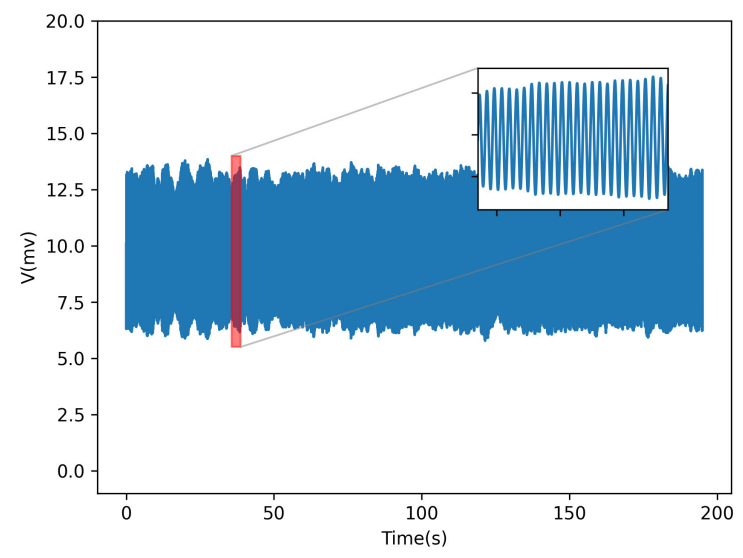

C

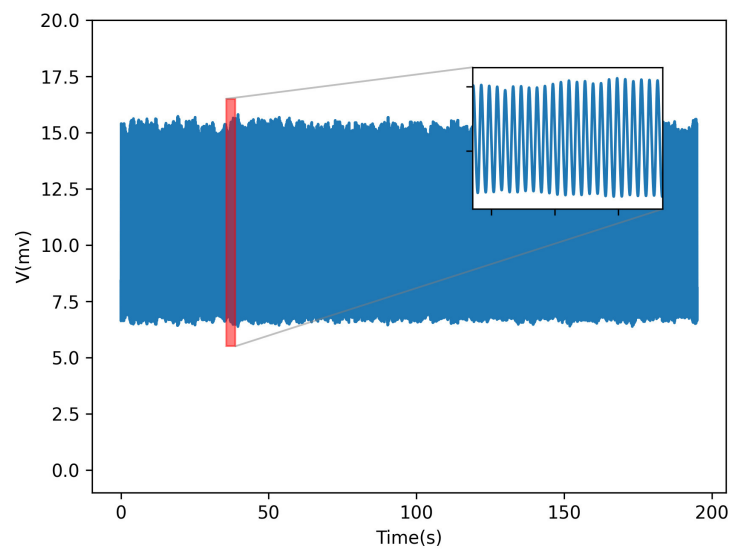

E

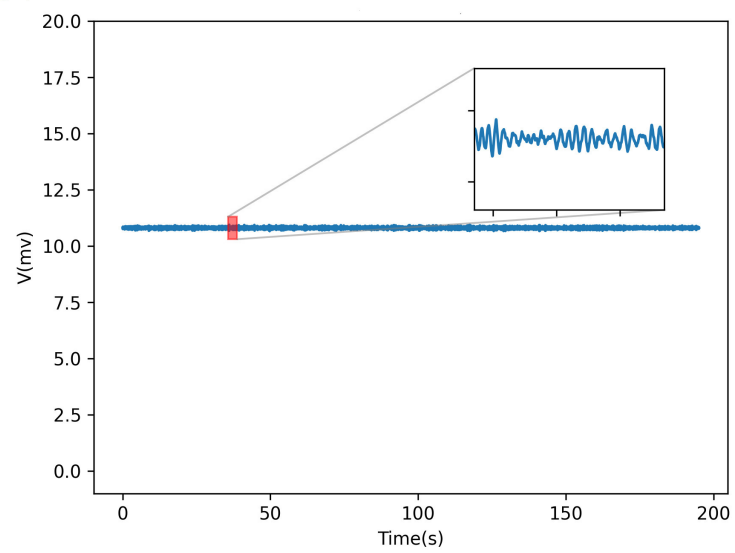

B

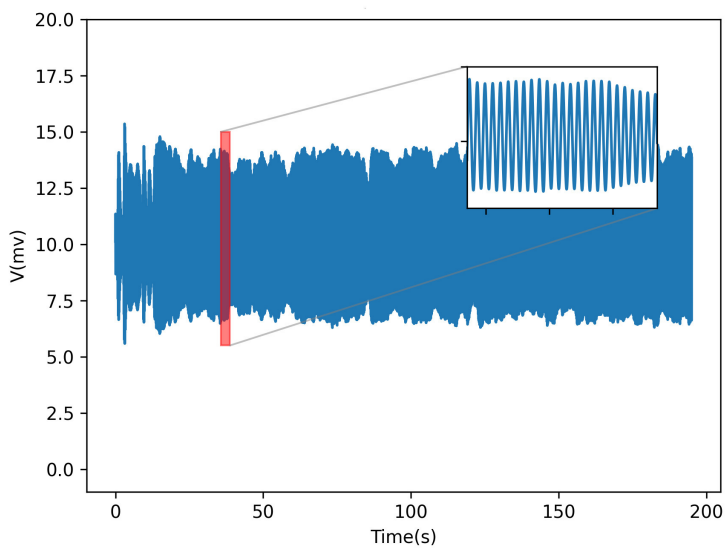

D

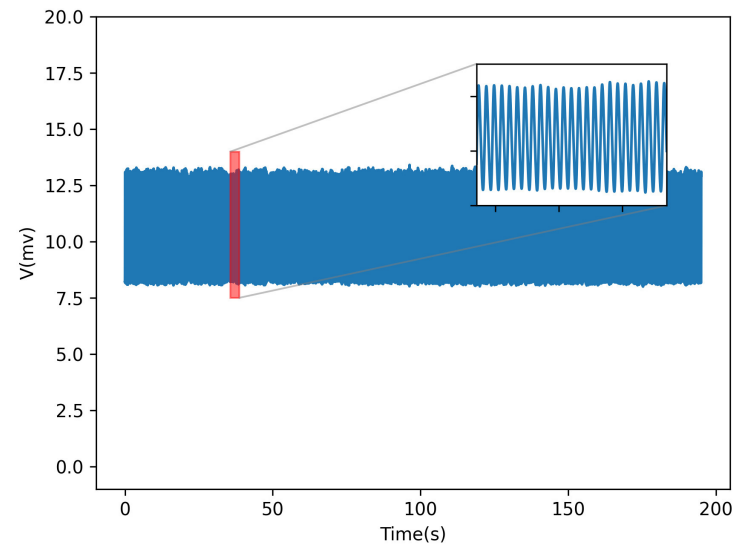

Figure 8. Time series of the mean of the network activity for $\alpha=3.5$ (A), 4 (B), 4.5 (C), 5 (D), and 5.5 (E). The inset of signals shows a specific segment of signal for 3 seconds. In $\alpha=5.5$, each node leaves the limit cycle and take in resting state (fixed-point). 
A

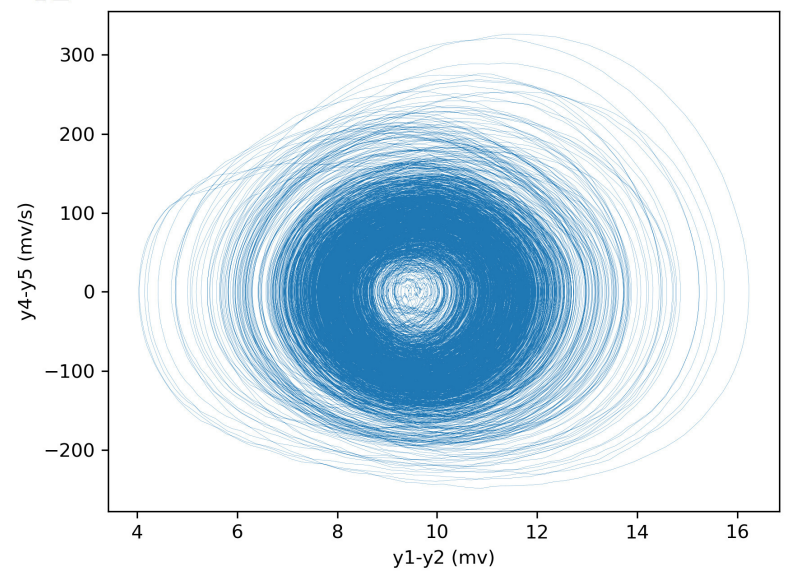

C

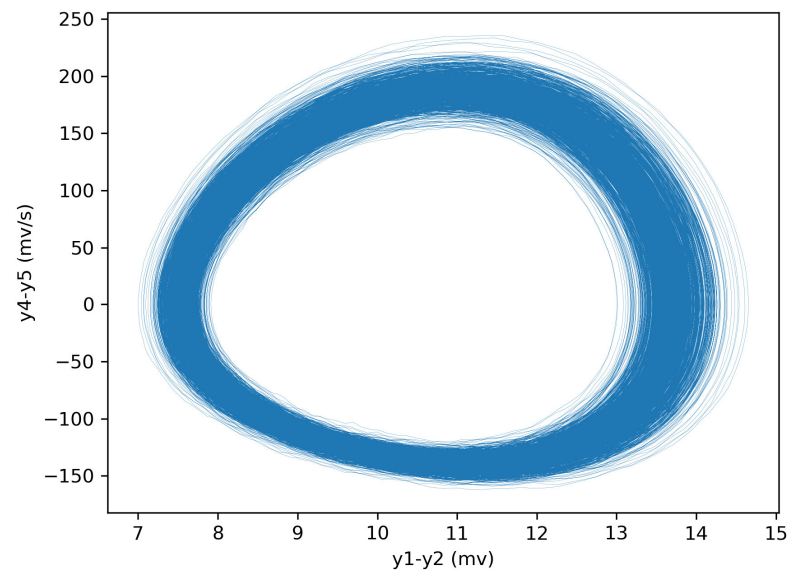

E

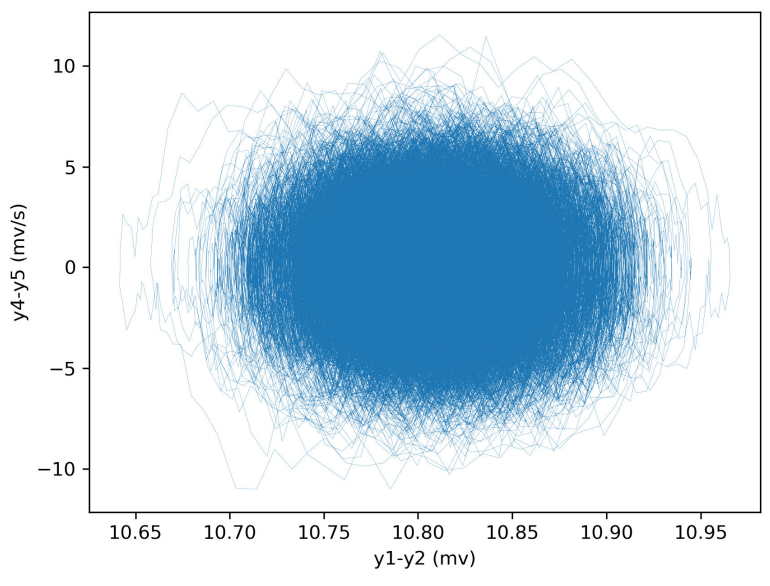

E

\section{B}

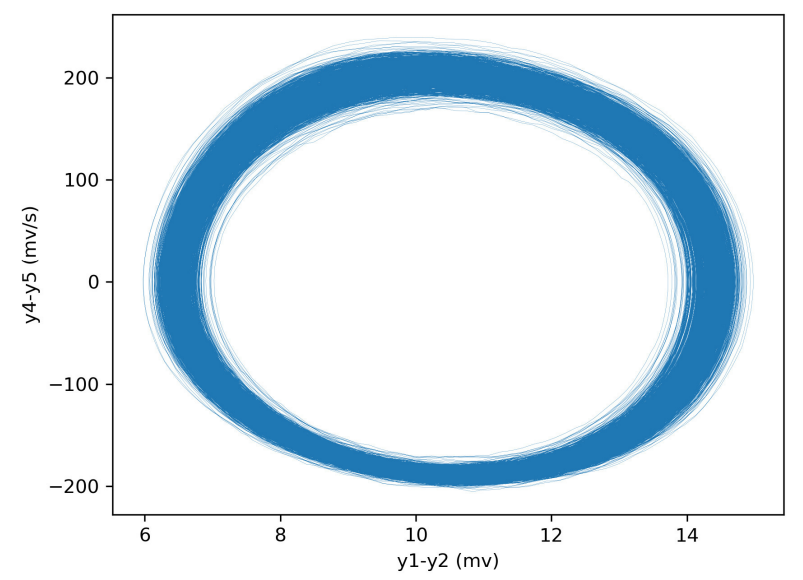

D

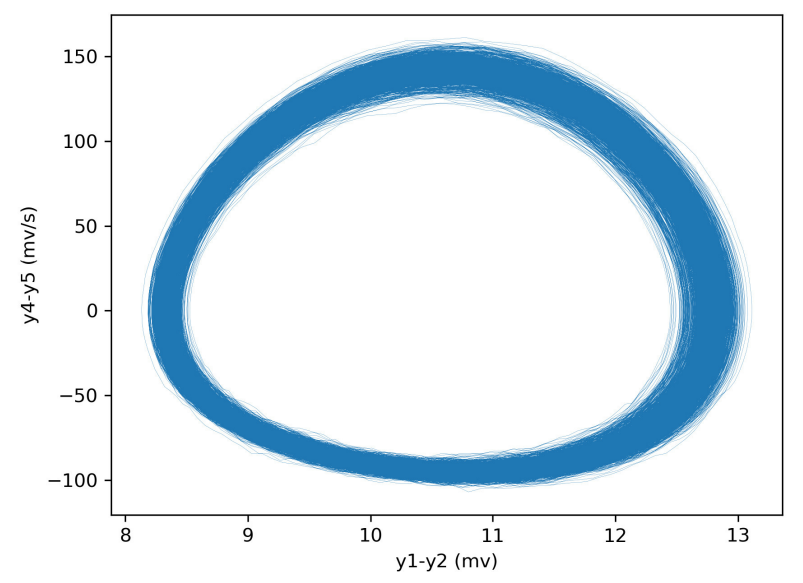


A

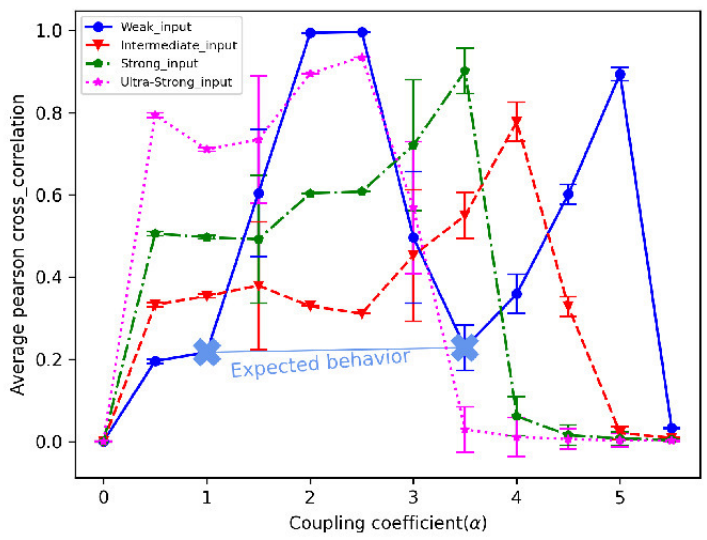

B

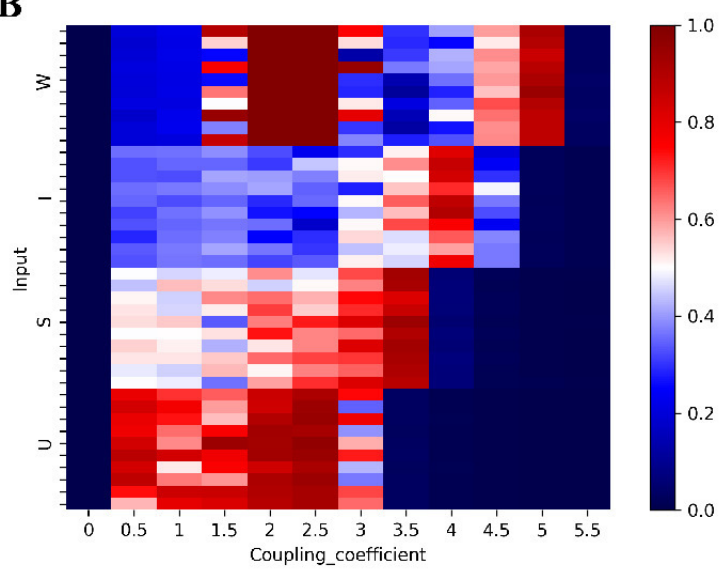

Figure 10. (A): The mean of the Pcc matrix versus coupling coefficient for four levels of input. Lower intensity input needs a larger coupling coefficient to reach its Pcc maximum. Expected behavior in the weak input level went forward as specified trend between two 'X' marks. (B): The heat-map of the mean of the Pcc matrix (W:weak, I:intermediate, S:strong, U:ultra-strong)

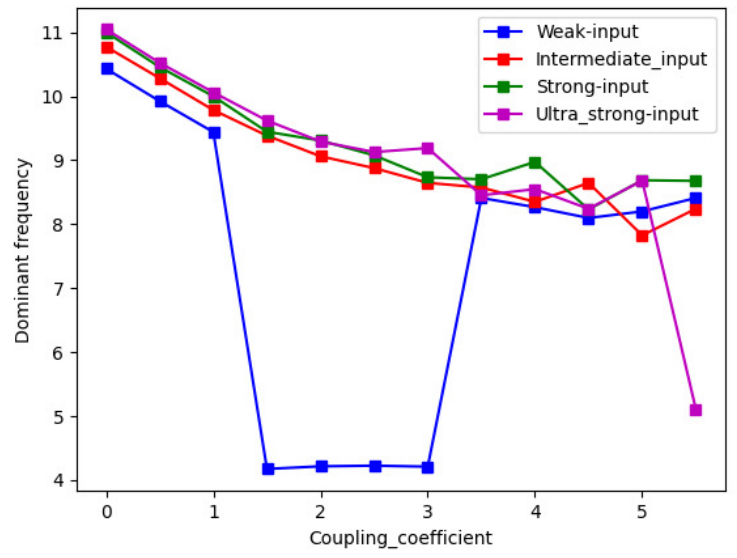

Figure 11. Dominant frequency as a function of the coupling coefficient. Increase coupling coefficient decreases the dominant frequency. 


\section{Power law and criticality}

It claimed that the normal brain acts in a critical regime ${ }^{47-50}$. It is an interesting and challenging question that how the critical dynamics can be detected in neural models. There are some measures of criticality that explain as follows.

The expression of the criticality hypothesis is that the brain may be taken on the edge of phase transition. We considered synchronization phase transition in our research, and so we study $\alpha=1.5,3$ candidate points that can be checked criticality on them. It is shown in ${ }^{75}$ that a different range of patterns can be generated by being on the edge of synchronization. Last two seconds of the spatiotemporal matrices in $\alpha=1,1.5,2,2.5,3$, and 3.5 are seen in figure 12 . In $\alpha=1$, the fluctuations are random, and the value of synchronization is low. Conversely, in alpha $=2$, the activity of the network is extremely ordered. This pattern is related to full synchronization that does not show a healthy state. Between these points, in $\alpha=1.5$, the network activity is among high and low synchrony. Similarly, in $\alpha=2.5$ and 3.5, activities are highly ordered and disordered, respectively, and in $\alpha=1.5$ and 3, the network shows a pattern between ordered and disordered states. So, it is possible to say these points (1.5 and 3) are critical. Another marker of criticality is the presence of high fluctuations. In critical points, the perturbations

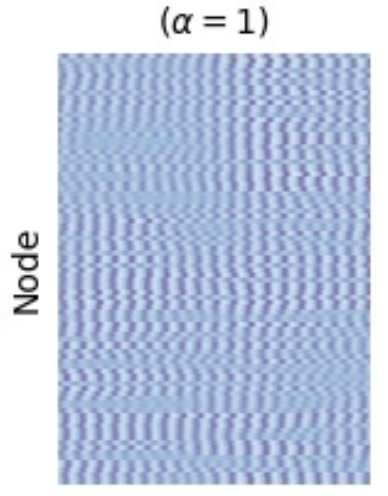

$(\alpha=2.5)$

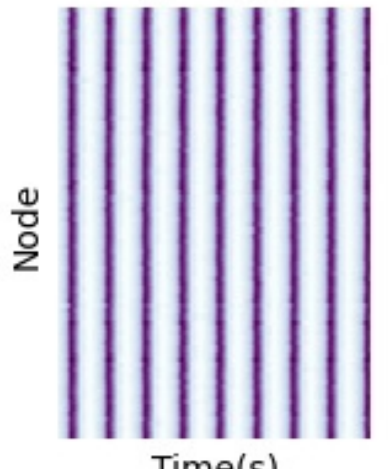

Time(s) $(\alpha=1.5)$

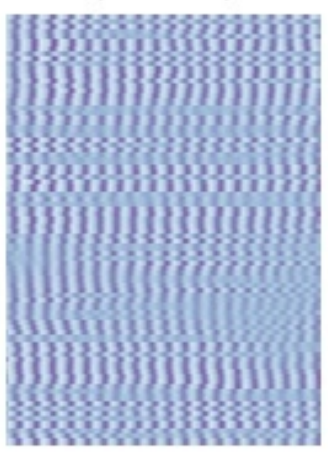

$(\alpha=3)$

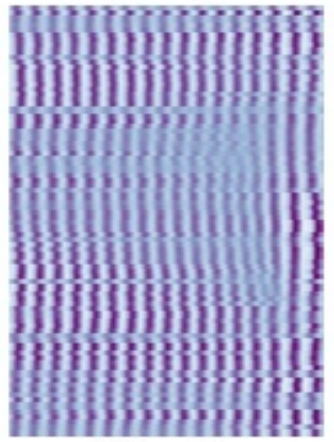

Time(s) $(\alpha=2)$

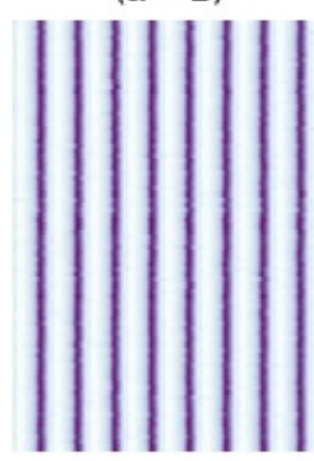

$(\alpha=3.5)$

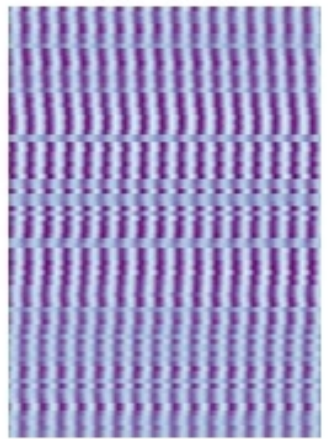

Time(s)

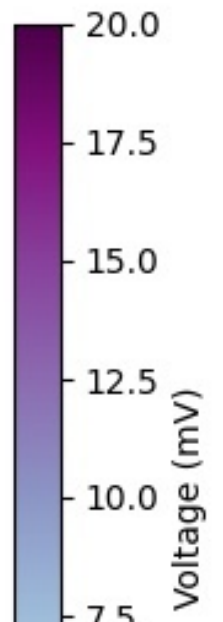

7.5

5.0

$-2.5$

0.0

Figure 12. The spatiotemporal matrices of the mean signal for different values of coupling coefficient. In $\alpha=1.5$ (3), there is a phase transition from disordered (ordered) to ordered (disordered) states, and the system in these coupling strengths is being on the edge of phase synchronization.

grow in magnitude, and different behavior occurs. Because the system stability is weak, it is expected to see events in each scale (micro or macro) that it is one of the most remarkable properties in criticality ${ }^{76,77}$. Broadly speaking, the border between scales is not clear. Based on figure $3, \alpha=1.5$ and 3 has more dispersion than other coupling strength. An extensive range of synchronization has just arisen in $\alpha=3$. Different patterns in the correlation matrices are seen in figure 7. In alpha=1.5, the dispersion of synchronization is approximately high, but the variety of schema fails here. Hence, alpha $=1.5$ may not be considered a critical point and does not have a plausible variation of observations.

Scale-invariant of neural avalanches is the most hallmark of criticality ${ }^{78}$. In phase transition points, the distinction between microscopic and macroscopic dynamics will be faded, i.e., neural avalanches start on the microscopic level and become so 
large until they cover overall dynamics and encompass the macroscopic scale. This event has corresponded to the powerlaw distribution of size and duration of avalanches ${ }^{79}$. We analyzed the squared Hilbert amplitude, which is a measure of instantaneous power. We set a threshold (mean of activity $(\theta)$ ), and we defined a neural avalanche size as the fluctuations of the integrated network mean activity when exceeds a threshold. Also, the duration of neural avalanches, i.e., the time between the start and end of a neural avalanche, is the length of time of the fluctuations stay above $\theta$. The distribution of size avalanches in the bifurcation points for one arbitrary realization in the weak input level is illustrated in figure 13. Although both coupling strengths can generate unusual events during the avalanche, the power-law behavior has not been seen in them. So based on this characteristic, these points are not critical. The presence of LRTCs in the amplitude of neural oscillations supports the critical

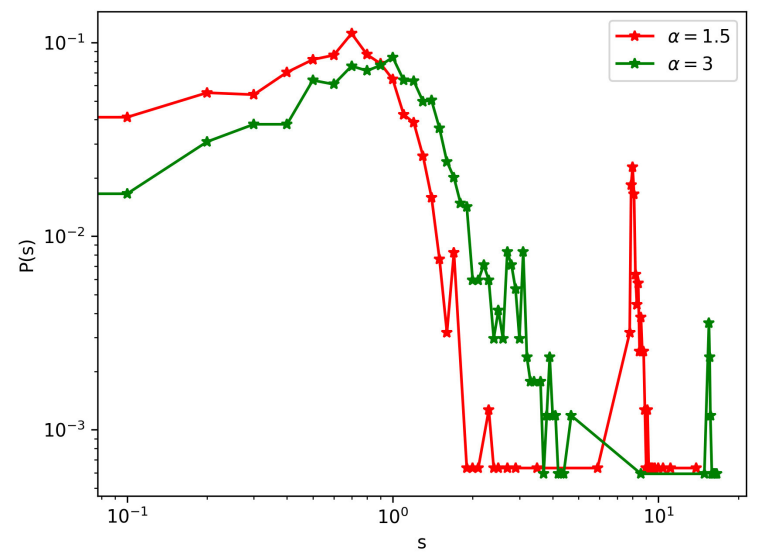

Figure 13. The distribution function of the size of avalanche in the weak input level at $\alpha=1.5,3$. Neither of them shows power-law behavior.

hypothesis. Indeed, long-range temporal correlations are a pivotal feature of criticality ${ }^{51,52}$.

The temporal correlation structures of the signal are investigated by the mean auto-correlation or DFA method. A signal can show LRTCs if its auto-correlation decays as a power law (with an exponent between -1 and 0). Generally, auto-correlation functions are very noisy in their tail, and so, the exponent estimation is very complicated. DFA is a proper technique that overcomes these problems ${ }^{52}$. This technique in neural mass models is used recently in ${ }^{35}$.

According to the previous section, it seems that one phase transition occurs in the weak input level around $\alpha=1.5,3$. We checked the presence of LRTCs at these points in an arbitrary repetition. Figure 14 shows the DFA applied in absolute signal Hilbert transform with no overlapping. The linear fit is not suitable for data (on the report of ${ }^{53}$ spline fit is the best fit model). So, LRTCs do not exist at these points. Similarly, LRTCs have not been exhibited in other coupling coefficients, and consequently, the network with defined parameters does not show power-law behavior and hence criticality due to this feature.

\section{Conclusion}

Our results showed that in a weak input level, the dominant frequency of a single unit and a network of units might be different. In ${ }^{55}$ Spiegler. et al. demonstrated that the dominant rhythm could be scaled by the ratio between the inhibitory and excitatory time constants. Although the alpha rhythm was prominent in a single node, the network made by single nodes not necessarily showed this rhythm. This consequence is an emergent property in a complex system ${ }^{80}$. i.e., each isolated node represents an alpha rhythm that can be changed when these units interact with each other in a connected network.

We explained elaborately that the input level and coupling coefficient were crucial parameters. Surprisingly, in the weak range of input, some interesting events occurred, e.g., an epileptic activity appeared that related to the first peak in the mean of the Pcc matrix and was unpredictable due to the plot's behavior. $\alpha=1.5,3$ were set as bifurcation points, and [1.5-3] was called "epileptic area". In $\alpha=1.5$, GSWDs (generalized spike-wave discharges) with approximately $4 \mathrm{~Hz}$ frequency band, were appeared. Commonly, GSWDs have arisen after paroxysmal oscillations in the corticothalamic networks but actually, their process is not clear yet ${ }^{81,82}$. Also, we observed that in an epileptic brain, increasing the coupling coefficient stimulates the network and nonlinear dynamics causes a jump between small and large amplitude oscillations. An important point to note is that different frequency bands have a remarkable role in epilepsy ${ }^{83}$. In $\alpha=1.5$, we discovered a transition from preictal to ictal state that is recognized by rhythmic spikes in a theta-band frequency and triggers the seizure activity. It is noteworthy the 
$\mathbf{A}$

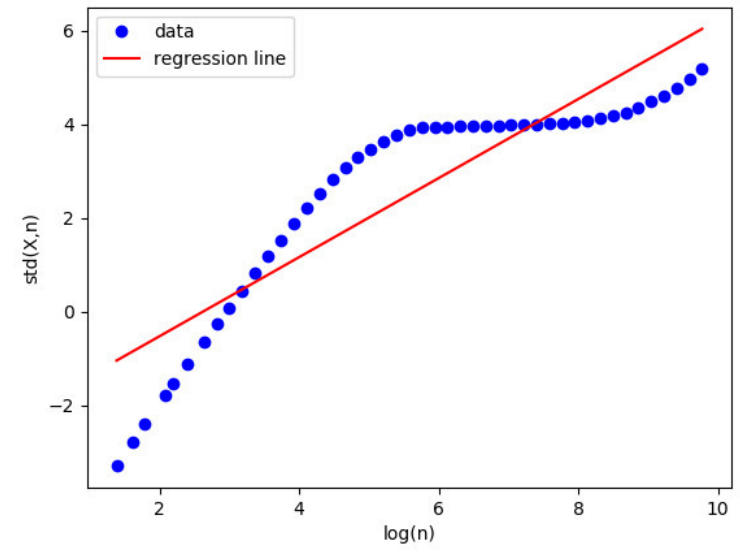

B

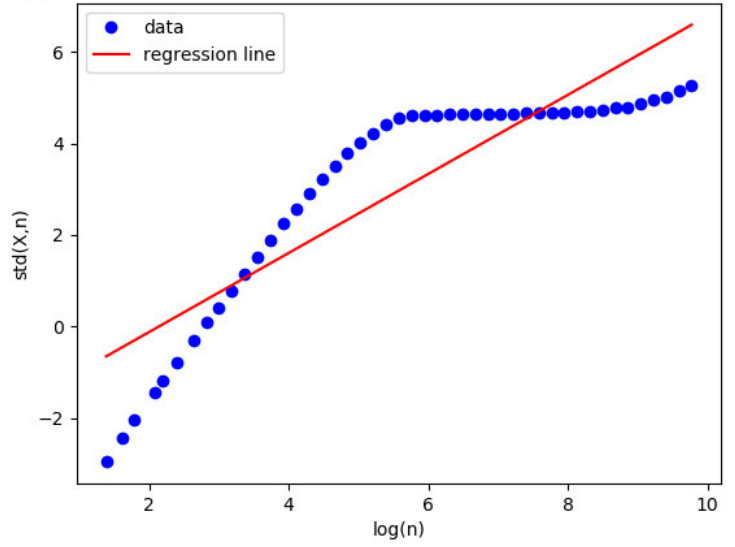

Figure 14. The fluctuation plot for $\alpha=1.5$ (A) and $\alpha=3(\mathbf{B})$ in the weak input level with the linear fit (red) in the log-log plot. This trend of data is piecewise linear and the linear fit is not suitable for them. So, LRTCs do not exist at these points.

frequency jump is a vital issue to comprehension epilepsy ${ }^{69,84}$.

Until now, all of these results are received from a weak input level. Although a high variance of the mean of the Pcc had shown in $\alpha=1.5$ and 3 in intermediate, strong, and ultra-strong input levels, coupling strength and input levels can not produce all behaviors in the weak input level, and so no bifurcation and phase transition exists in these cases.

In the final section, we presented some markers of criticality and investigated them on bifurcation points. Results showed a number of the criticality indicators are held in these points and others not. i.e., at the $\alpha=1.5$ and 3, based on some measures system shows signatures of criticality, and based on some other measures, it does not. Indeed, there is a difference in critical conception in the brain and the statistical mechanics. A critical point in the brain can extend to a critical area, Griffith phase ${ }^{85}$, and criticality is not all or nothing attitude. Moreover, our research has not proven the accept or reject the critical hypothesis. It seems that the Jansen-Rits model has a percentage of criticality theory.

As a summary, the randomness of input, initial conditions, and coupling strengths in a network can generate different complex behaviors. We also should mention these behaviors are not just grounds to triggers the seizure activity because understanding the epilepsy dynamics can not be achieved easily.

It seems that the delay between the neural populations in networks can produce complex dynamics, and in this study is assumed to be zero. The findings have been reported by ${ }^{35,86}$ were considered the delay in other neural mass models.

Notice, this model is analyzed in the alpha frequency band. It means that the coupling between excitatory and inhibitory mass in each node was set to 135 . Future reports can focus on the epilepsy state, and their results can be effective in the detection and treatment of this disease.

Changing the standard deviation of stochastic input may have a significant effect that was discarded here, and maybe some interesting results will be achieved by considering this.

Criticality is an asserted assumption in a normal brain. The results in this paper show that the criticality hypothesis is not all or nothing theory. The Jansen-Rit dynamic as a neural mass model with assumed parameters does not have all critical features, and we can only admit that they have a percentage of criticality. We are left with the question, whether the Jansen-Rit model can be modified to show all markers of critical behavior and, if it is possible, adjusting which parameter (noise, delay, coupling between inhibitory ensembles, etc.) do it.

\section{References}

1. Yamada, T. \& Fujisaka, H. Stability theory of synchronized motion in coupled-oscillator systems. ii: The mapping approach. Prog. Theor. Phys. 70, 1240-1248 (1983).

2. Rosenblum, M. G., Pikovsky, A. S. \& Kurths, J. Phase synchronization of chaotic oscillators. Phys. review letters 76, 1804 (1996).

3. Fries, P. A mechanism for cognitive dynamics: neuronal communication through neuronal coherence. Trends cognitive sciences 9, 474-480 (2005). 
4. Siegel, M., Donner, T. H. \& Engel, A. K. Spectral fingerprints of large-scale neuronal interactions. Nat. Rev. Neurosci. 13, 121-134 (2012).

5. Fries, P. Rhythms for cognition: communication through coherence. Neuron 88, 220-235 (2015).

6. Bastos, A. M. \& Schoffelen, J.-M. A tutorial review of functional connectivity analysis methods and their interpretational pitfalls. Front. systems neuroscience 9,175 (2016).

7. Castanedo-Guerra, I., Steur, E. \& Nijmeijer, H. Synchronization of "light-sensitive" hindmarsh-rose neurons. Commun. Nonlinear Sci. Numer. Simul. 57, 322-330 (2018).

8. Liu, Y., Xu, Y. \& Ma, J. Synchronization and spatial patterns in a light-dependent neural network. Commun. Nonlinear Sci. Numer. Simul. 89, 105297 (2020).

9. Bashkirtseva, I., Ryashko, L. \& Pisarchik, A. N. Stochastic transitions between in-phase and anti-phase synchronization in coupled map-based neural oscillators. Commun. Nonlinear Sci. Numer. Simul. 95, 105611 (2021).

10. Wang, H., Wang, Q. \& Lu, Q. Bursting oscillations, bifurcation and synchronization in neuronal systems. Chaos, Solitons \& Fractals 44, 667-675 (2011).

11. Roelfsema, P. R., Engel, A. K., König, P. \& Singer, W. Visuomotor integration is associated with zero time-lag synchronization among cortical areas. Nature 385, 157-161 (1997).

12. Mizuhara, H. \& Yamaguchi, Y. Human cortical circuits for central executive function emerge by theta phase synchronization. Neuroimage 36, 232-244 (2007).

13. Uhlhaas, P. J. \& Singer, W. Neural synchrony in brain disorders: relevance for cognitive dysfunctions and pathophysiology. neuron 52, 155-168 (2006).

14. Schnitzler, A. \& Gross, J. Normal and pathological oscillatory communication in the brain. Nat. reviews neuroscience 6, 285-296 (2005).

15. Li, X., Yao, X., Fox, J. \& Jefferys, J. G. Interaction dynamics of neuronal oscillations analysed using wavelet transforms. J. neuroscience methods 160, 178-185 (2007).

16. Aviyente, S., Bernat, E. M., Evans, W. S. \& Sponheim, S. R. A phase synchrony measure for quantifying dynamic functional integration in the brain. Tech. Rep., Wiley Online Library (2011).

17. Zheng, Y., Wang, G., Li, K., Bao, G. \& Wang, J. Epileptic seizure prediction using phase synchronization based on bivariate empirical mode decomposition. Clin. Neurophysiol. 125, 1104-1111 (2014).

18. Budzinski, R., Boaretto, B., Prado, T. \& Lopes, S. Phase synchronization and intermittent behavior in healthy and alzheimer-affected human-brain-based neural network. Phys. Rev. E 99, 022402 (2019).

19. Ahmadlou, M., Adeli, H. \& Adeli, A. Fuzzy synchronization likelihood-wavelet methodology for diagnosis of autism spectrum disorder. J. neuroscience methods 211, 203-209 (2012).

20. Bob, P., Palus, M., Susta, M. \& Glaslova, K. Eeg phase synchronization in patients with paranoid schizophrenia. Neurosci. letters 447, 73-77 (2008).

21. Ngugi, A. K., Bottomley, C., Kleinschmidt, I., Sander, J. W. \& Newton, C. R. Estimation of the burden of active and life-time epilepsy: a meta-analytic approach. Epilepsia 51, 883-890 (2010).

22. Penfield, W. \& Jasper, H. Epilepsy and the functional anatomy of the human brain. (Little, Brown \& Co., 1954).

23. Buzsaki, G. Rhythms of the Brain (Oxford University Press, 2006).

24. Nowotny, T., Huerta, R. \& Rabinovich, M. I. Neuronal synchrony: peculiarity and generality. Chaos: An Interdiscip. J. Nonlinear Sci. 18, 5692 (2008).

25. Ryashko, L. \& Slepukhina, E. Noise-induced toroidal excitability in neuron model. Commun. Nonlinear Sci. Numer. Simul. 82, $105071(2020)$.

26. Kelso, J. S. Dynamic patterns: The self-organization of brain and behavior (MIT press, 1997).

27. Deco, G., Jirsa, V. K., Robinson, P. A., Breakspear, M. \& Friston, K. The dynamic brain: from spiking neurons to neural masses and cortical fields. PLoS Comput. Biol 4, e1000092 (2008).

28. Coombes, S. \& Byrne, Á. Next generation neural mass models. In Nonlinear dynamics in computational neuroscience, 1-16 (Springer, 2019).

29. Breakspear, M. Dynamic models of large-scale brain activity. Nat. neuroscience 20, 340-352 (2017). 
30. Freeman, W. J. Simulation of chaotic eeg patterns with a dynamic model of the olfactory system. Biol. cybernetics $\mathbf{5 6}$, 139-150 (1987).

31. Chang, H.-J. \& Freeman, W. J. Parameter optimization in models of the olfactory neural system. Neural Networks 9, 1-14 (1996).

32. Anitta, F., Sunitha, R., Pradhan, N. \& Sreedevi, A. Non-linear analysis of time series generated from the freeman k-set model. In Cognitive Informatics and Soft Computing, 217-225 (Springer, 2019).

33. Wong, K.-F. \& Wang, X.-J. A recurrent network mechanism of time integration in perceptual decisions. J. Neurosci. 26, 1314-1328 (2006).

34. Deco, G. et al. Resting-state functional connectivity emerges from structurally and dynamically shaped slow linear fluctuations. J. Neurosci. 33, 11239-11252 (2013).

35. Daffertshofer, A., Ton, R., Pietras, B., Kringelbach, M. L. \& Deco, G. Scale-freeness or partial synchronization in neural mass phase oscillator networks: Pick one of two? NeuroImage 180, 428-441 (2018).

36. Kashyap, A. \& Keilholz, S. Dynamic properties of simulated brain network models and empirical resting-state data. Netw. Neurosci. 3, 405-426 (2019).

37. Khoshkhou, M. \& Montakhab, A. Spike-timing-dependent plasticity with axonal delay tunes networks of izhikevich neurons to the edge of synchronization transition with scale-free avalanches. Front. systems neuroscience 13, 73 (2019).

38. Nazemi, P. S. \& Jamali, Y. On the influence of structural connectivity on the correlation patterns and network synchronization. Front. Comput. Neurosci. 12, 105 (2019).

39. Budzinski, R. C., Boaretto, B. R. R., Prado, T. L. \& Lopes, S. R. Investigation of details in the transition to synchronization in complex networks by using recurrence analysis. Math. Comput. Appl. 24, 42 (2019).

40. Budzinski, R., Boaretto, B., Prado, T. \& Lopes, S. Synchronization domains in two coupled neural networks. Commun. Nonlinear Sci. Numer. Simul. 75, 140-151 (2019).

41. Forrester, M., Crofts, J. J., Sotiropoulos, S. N., Coombes, S. \& O’Dea, R. D. The role of node dynamics in shaping emergent functional connectivity patterns in the brain. Netw. Neurosci. 4, 467-483 (2020).

42. van Rotterdam, A., Da Silva, F. L., Van den Ende, J., Viergever, M. \& Hermans, A. A model of the spatial-temporal characteristics of the alpha rhythm. Bull. mathematical biology 44, 283-305 (1982).

43. Freeman, W. J. Simulation of chaotic eeg patterns with a dynamic model of the olfactory system. Biol. cybernetics $\mathbf{5 6}$, 139-150 (1987).

44. Braitenberg, V. \& Schüz, A. Cortex: statistics and geometry of neuronal connectivity (Springer Science \& Business Media, 2013).

45. Lachaux, J.-P., Rodriguez, E., Martinerie, J. \& Varela, F. J. Measuring phase synchrony in brain signals. Hum. brain mapping 8, 194-208 (1999).

46. Peng, C.-K. et al. Mosaic organization of dna nucleotides. Phys. review e 49, 1685 (1994).

47. Beggs, J. M. \& Plenz, D. Neuronal avalanches in neocortical circuits. J. neuroscience 23, 11167-11177 (2003).

48. Kitzbichler, M. G., Smith, M. L., Christensen, S. R. \& Bullmore, E. Broadband criticality of human brain network synchronization. PLoS Comput. Biol 5, e1000314 (2009).

49. Tetzlaff, C., Okujeni, S., Egert, U., Wörgötter, F. \& Butz, M. Self-organized criticality in developing neuronal networks. PLoS Comput. Biol 6, e1001013 (2010).

50. Meisel, C., Storch, A., Hallmeyer-Elgner, S., Bullmore, E. \& Gross, T. Failure of adaptive self-organized criticality during epileptic seizure attacks. PLoS Comput. Biol 8, e1002312 (2012).

51. Linkenkaer-Hansen, K., Nikouline, V. V., Palva, J. M. \& Ilmoniemi, R. J. Long-range temporal correlations and scaling behavior in human brain oscillations. J. Neurosci. 21, 1370-1377 (2001).

52. Hardstone, R. et al. Detrended fluctuation analysis: a scale-free view on neuronal oscillations. Front. physiology 3, 450 (2012).

53. Botcharova, M., Farmer, S. \& Berthouze, L. A maximum likelihood based technique for validating detrended fluctuation analysis (ml-dfa). arXiv: Quant. Methods (2013).

54. Grimbert, F. \& Faugeras, O. Bifurcation analysis of jansen's neural mass model. Neural computation 18, 3052-3068 (2006). 
55. Spiegler, A., Kiebel, S. J., Atay, F. M. \& Knösche, T. R. Bifurcation analysis of neural mass models: Impact of extrinsic inputs and dendritic time constants. NeuroImage 52, 1041-1058 (2010).

56. Kameneva, T., Ying, T., Guo, B. \& Freestone, D. R. Neural mass models as a tool to investigate neural dynamics during seizures. J. computational neuroscience 42, 203-215 (2017).

57. Jansen, B. H. \& Rit, V. G. Electroencephalogram and visual evoked potential generation in a mathematical model of coupled cortical columns. Biol. cybernetics 73, 357-366 (1995).

58. Touboul, J., Wendling, F., Chauvel, P. \& Faugeras, O. Neural mass activity, bifurcations, and epilepsy. Neural computation 23, 3232-3286 (2011).

59. Ableidinger, M., Buckwar, E. \& Hinterleitner, H. A stochastic version of the jansen and rit neural mass model: Analysis and numerics. The J. Math. Neurosci. 7, 1-35 (2017).

60. Ahmadizadeh, S. et al. Bifurcation analysis of two coupled jansen-rit neural mass models. PloS one 13, e0192842 (2018).

61. Aburn, M. J., Holmes, C., Roberts, J. A., Boonstra, T. W. \& Breakspear, M. Critical fluctuations in cortical models near instability. Front. physiology 3, 331 (2012).

62. Stefanovski, L. et al. Linking molecular pathways and large-scale computational modeling to assess candidate disease mechanisms and pharmacodynamics in alzheimer's disease. Front. computational neuroscience 13, 54 (2019).

63. Watts, D. J. \& Strogatz, S. H. Collective dynamics of 'small-world'networks. nature 393, 440-442 (1998).

64. Daffertshofer, A. \& van Wijk, B. On the influence of amplitude on the connectivity between phases. Front. neuroinformatics 5, 6 (2011).

65. Ódor, G. \& Kelling, J. Critical synchronization dynamics of the kuramoto model on connectome and small world graphs. Sci. reports 9, 1-10 (2019).

66. Frolov, N. et al. Chimera-like behavior in a heterogeneous kuramoto model: The interplay between attractive and repulsive coupling. Chaos: An Interdiscip. J. Nonlinear Sci. 30, 081102 (2020).

67. Rüemelin, W. Numerical treatment of stochastic differential equations. SIAM J. on Numer. Analysis 19, 604-613 (1982).

68. Breakspear, M. et al. A unifying explanation of primary generalized seizures through nonlinear brain modeling and bifurcation analysis. Cereb. Cortex 16, 1296-1313 (2006).

69. Haghighi, H. S. \& Markazi, A. A new description of epileptic seizures based on dynamic analysis of a thalamocortical model. Sci. reports 7, 1-10 (2017).

70. Maturana, M. I. et al. Critical slowing down as a biomarker for seizure susceptibility. Nat. communications 11, 1-12 (2020).

71. Paula, C. A. R. et al. High-frequency eeg variations in children with autism spectrum disorder during human faces visualization. BioMed research international 2017 (2017).

72. Igberaese, A. E. \& Tcheslavski, G. V. Eeg power spectrum as a biomarker of autism: a pilot study. Int. J. Electron. Healthc. 10, 275-286 (2018).

73. Fan, D., Liu, S. \& Wang, Q. Stimulus-induced epileptic spike-wave discharges in thalamocortical model with disinhibition. Sci. reports 6, 1-21 (2016).

74. Deeba, F., Sanz-Leon, P. \& Robinson, P. Unified dynamics of interictal events and absence seizures. Phys. Rev. E 100, 022407 (2019).

75. Khoshkhou, M. \& Montakhab, A. Beta-rhythm oscillations and synchronization transition in network models of izhikevich neurons: effect of topology and synaptic type. Front. computational neuroscience 12, 59 (2018).

76. Hesse, J. \& Gross, T. Self-organized criticality as a fundamental property of neural systems. Front. systems neuroscience 8, 166 (2014).

77. Cocchi, L., Gollo, L. L., Zalesky, A. \& Breakspear, M. Criticality in the brain: A synthesis of neurobiology, models and cognition. Prog. neurobiology 158, 132-152 (2017).

78. Beggs, J. M. \& Plenz, D. Neuronal avalanches in neocortical circuits. J. neuroscience 23, 11167-11177 (2003).

79. Levy, M. \& Solomon, S. Power laws are logarithmic boltzmann laws. Int. J. Mod. Phys. C 7, 595-601 (1996).

80. Johnson, C. A handbook of incident and accident reporting. Cité dans la 115 (2003). 
81. van Luijtelaar, G. \& Sitnikova, E. Global and focal aspects of absence epilepsy: the contribution of genetic models. Neurosci. \& Biobehav. Rev. 30, 983-1003 (2006).

82. Bernhardt, B. C. et al. Thalamo-cortical network pathology in idiopathic generalized epilepsy: insights from mri-based morphometric correlation analysis. Neuroimage 46, 373-381 (2009).

83. Blume, W. T., Young, G. B. \& Lemieux, J. F. Eeg morphology of partial epileptic seizures. Electroencephalogr. clinical neurophysiology 57, 295-302 (1984).

84. Suffczynski, P., Kalitzin, S. \& Da Silva, F. L. Dynamics of non-convulsive epileptic phenomena modeled by a bistable neuronal network. Neuroscience 126, 467-484 (2004).

85. Moretti, P. \& Muñoz, M. A. Griffiths phases and the stretching of criticality in brain networks. Nat. communications 4, $1-10$ (2013).

86. Conti, F. \& Van Gorder, R. A. The role of network structure and time delay in a metapopulation wilson-cowan model. $J$. theoretical biology 477, 1-13 (2019).

\section{Acknowledgements}

The second author is indebted to the Research Core: "Bio-Mathematics with computational approach" of Tarbiat Modares University, with Grant No. IG-39706. This work has been supported in part by a grant from the Cognitive Sciences and Technologies Council with grant No. 8226.

\section{Author contributions statement}

Yousef Jamali: Supervision, Conceptualization, Formal analysis, Investigation, Methodology, Project administration, Resources, Software, Validation, Writing - review and editing

Sheida Kazemi: Conceptualization, Data curation, Formal analysis, Investigation, Methodology, Software, Validation, Visualization, Writing - original draft, Writing - review and editing

\section{Competing Interests Statement}

The authors declare no competing financial interests. 
Figures

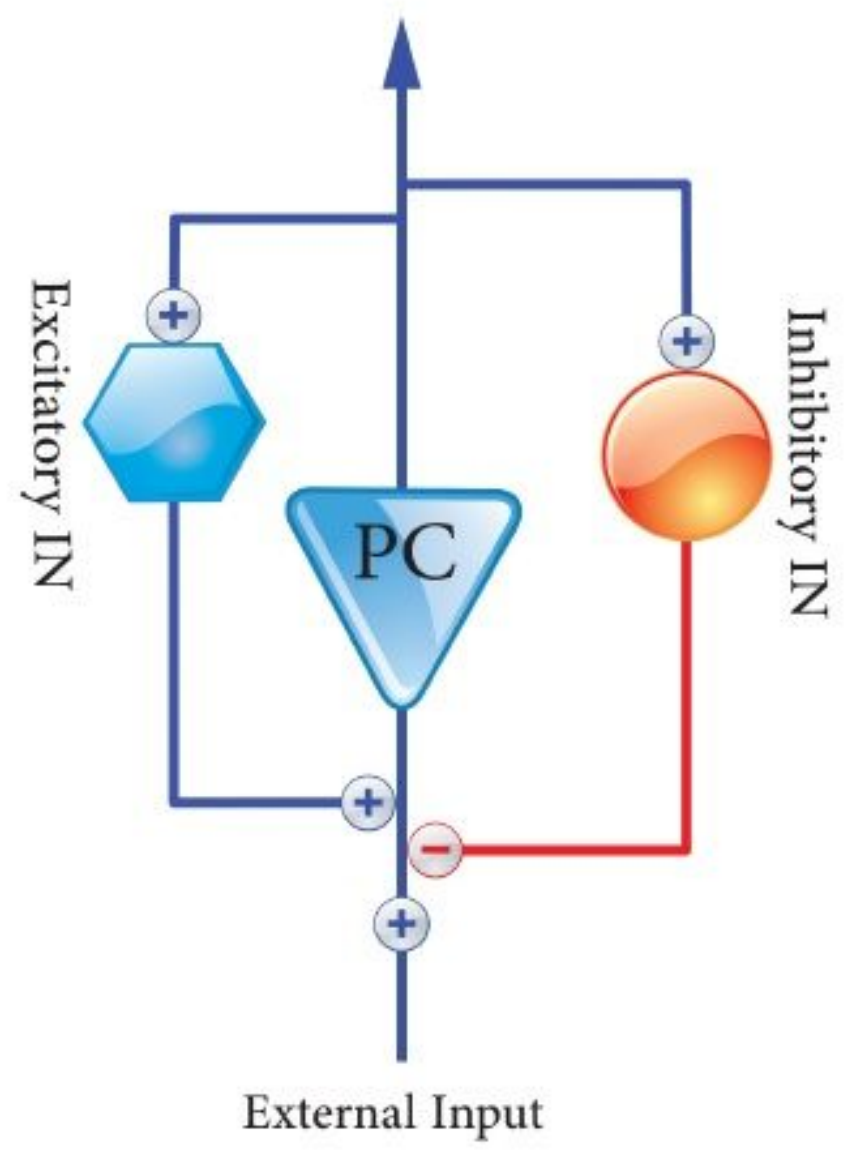

Figure 1

Schematic representation of Jansen-Rit model. A main population (pyramidal cell) interacts with two populations: excitatory interneuron (left) and inhibitory interneuron (right). 


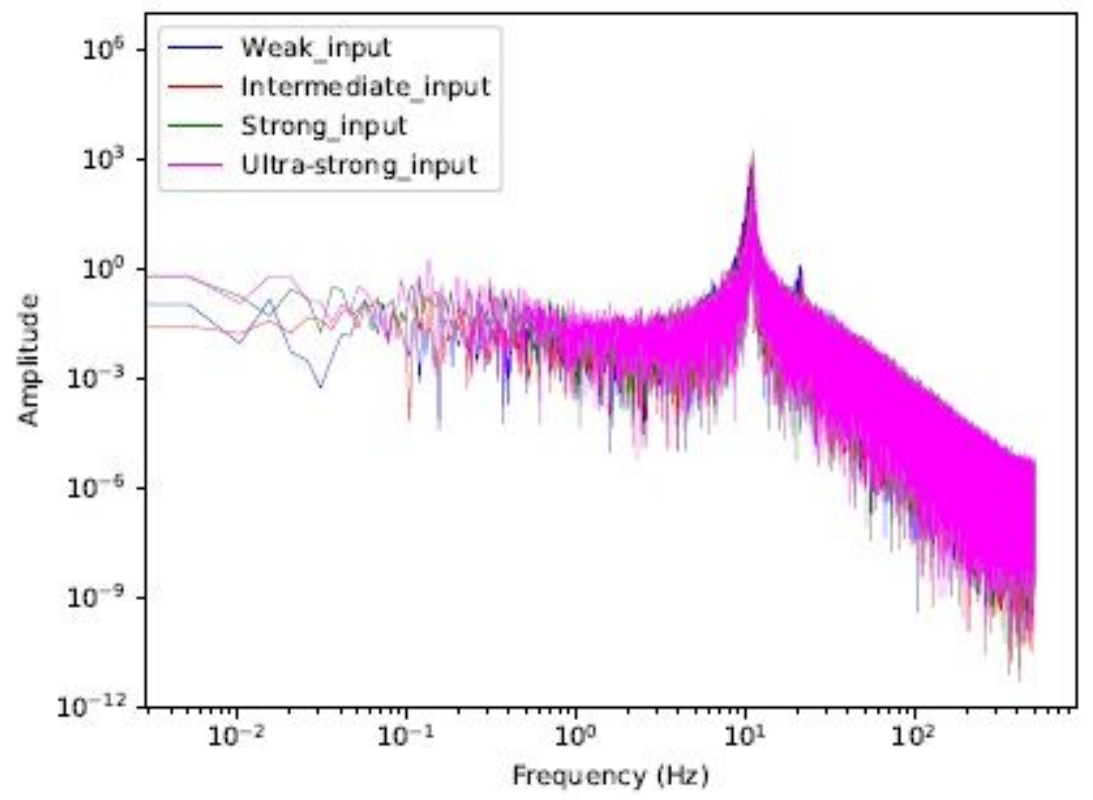

Figure 2

Power spectrum for a single unit in different level inputs. Alpha rhythm is dominant in all of them.

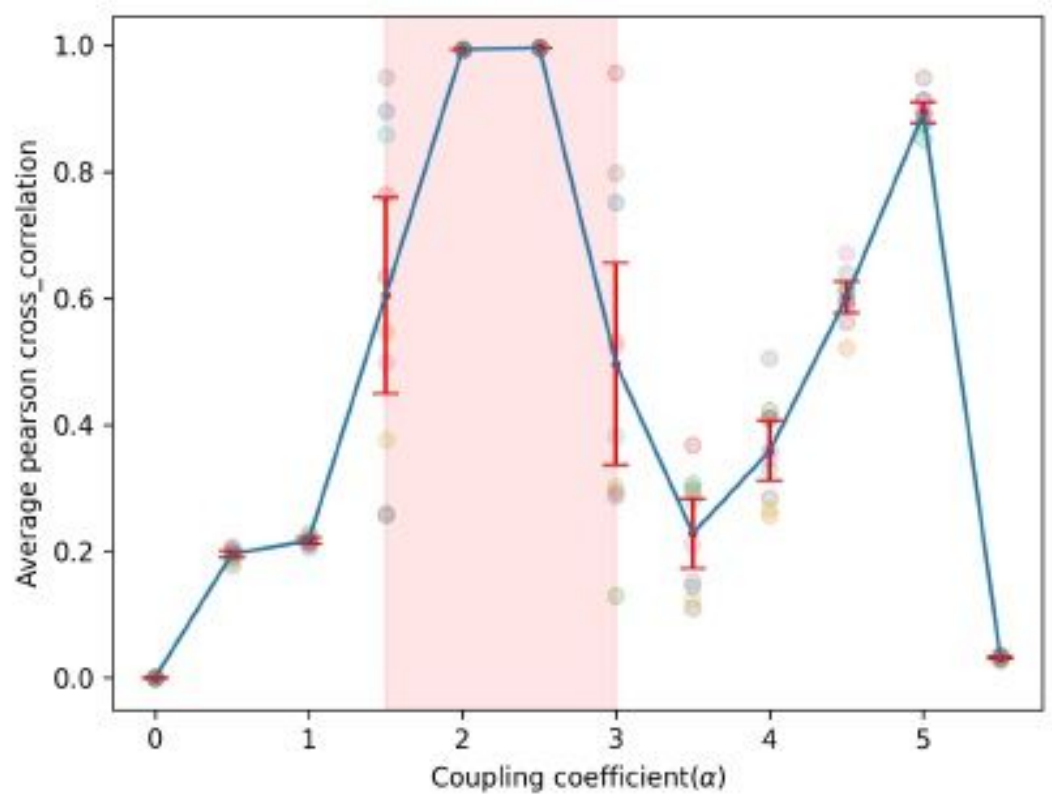

Figure 3

Mean of the Pearson cross-correlation matrix according to the coupling coefficient in a weak input area. In $\mathrm{a}=1.5$ and 3 , the dispersion of the mean of the Pcc are high and the area between them is related to the epilepsy regime. 
A

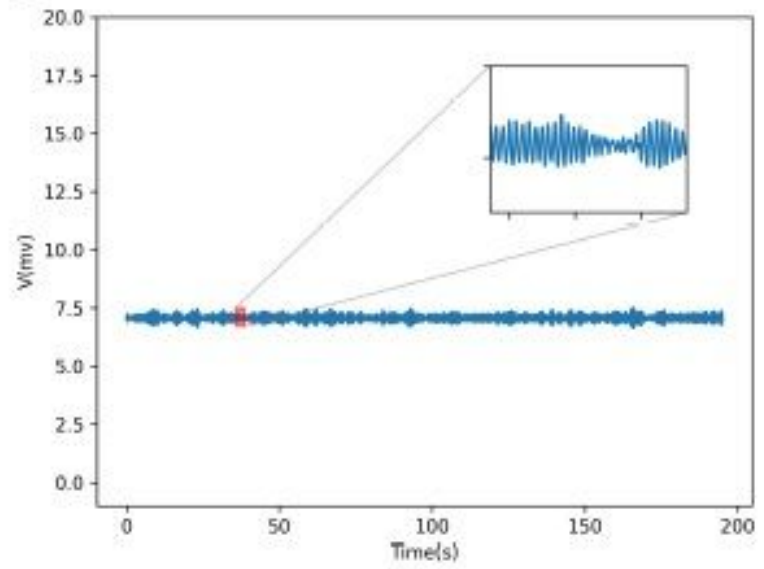

C

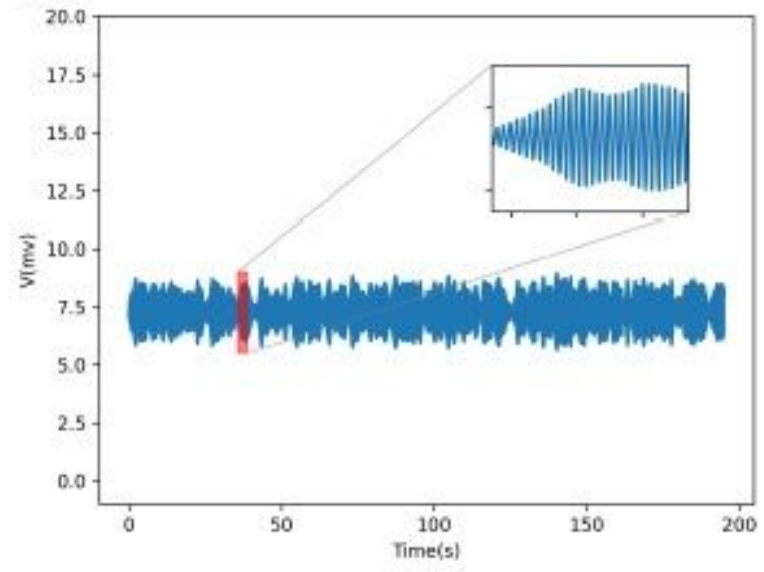

$\mathbf{E}$

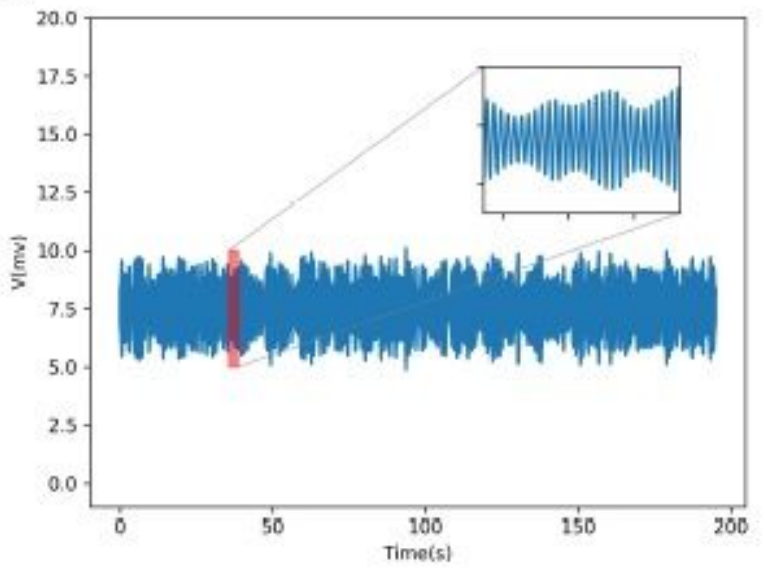

B

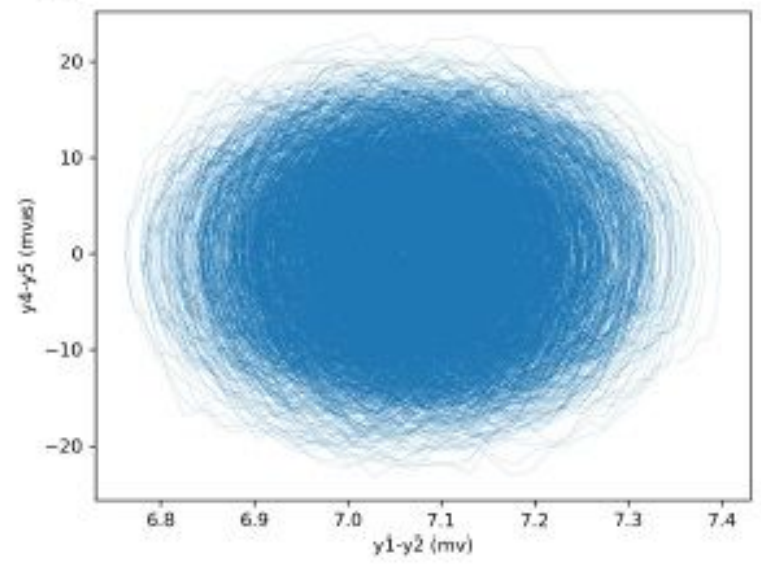

D
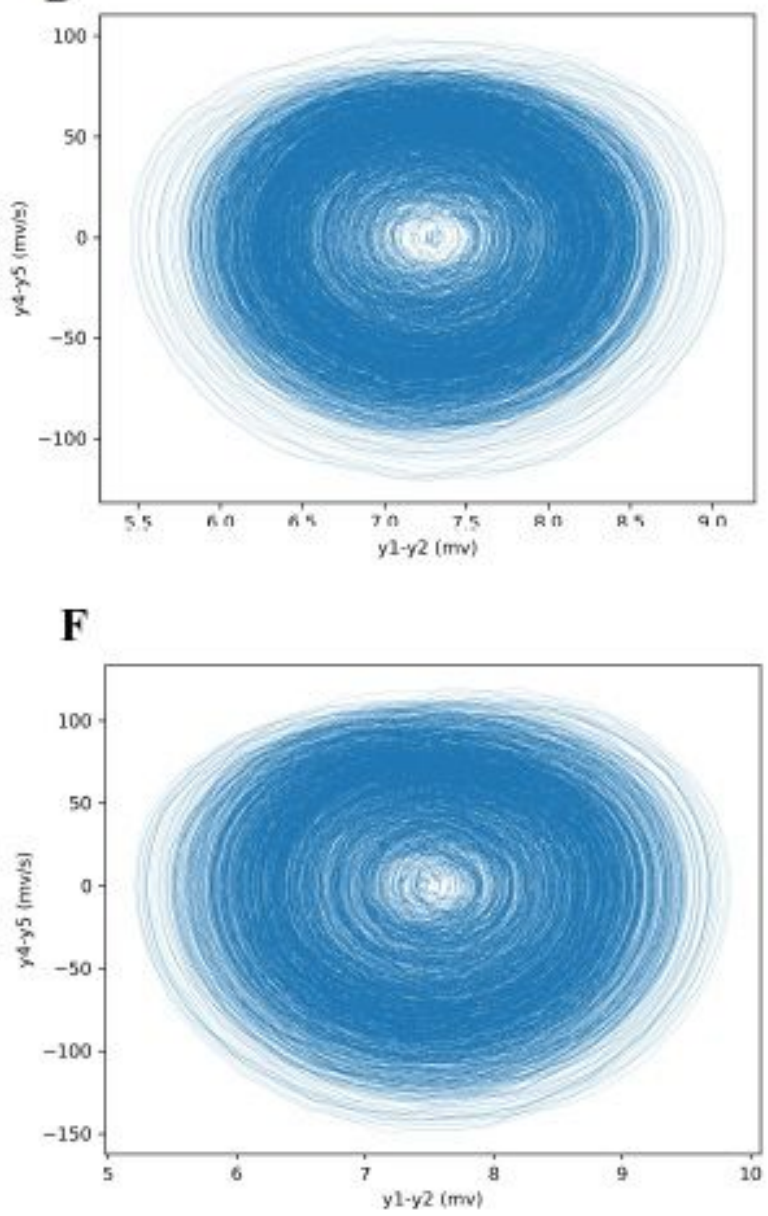

\section{Figure 4}

Time series of the mean of the network activity for $a=0(A), 0.5(C)$, and $1(E)$ and their phase space $(B$, $D, F)$. The $x$-axis and $y$-axis show the output signal and its derivation, respectively. By increasing the coupling coefficient, the mean signal amplitude gets larger. The inset of (A), (C) and (E) shows a specific segment of signal for 3 seconds. 
A

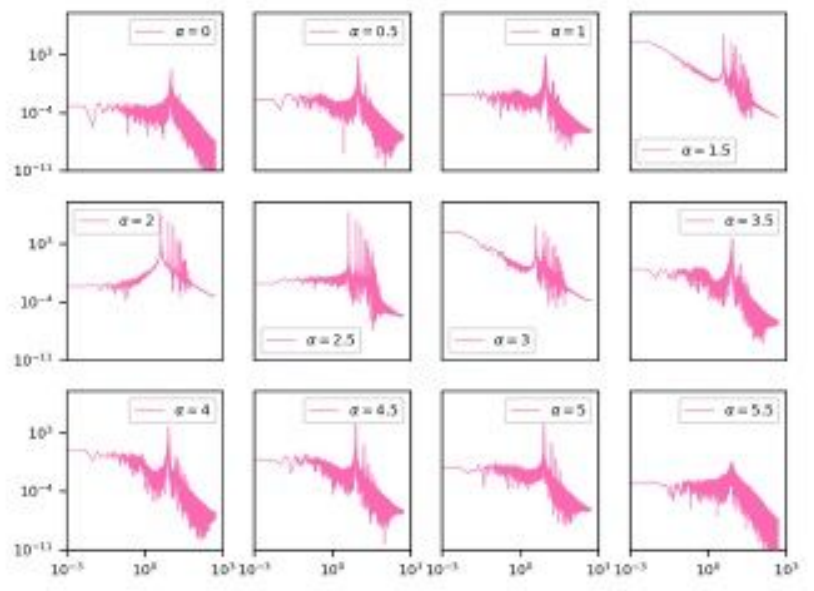

B

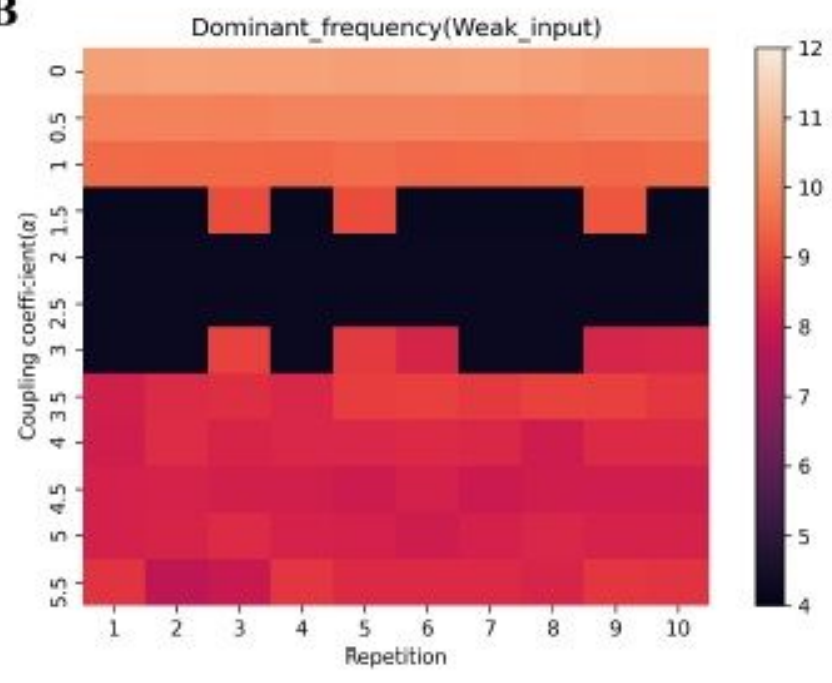

Figure 5

(A): Power spectrum for different coupling coefficients in weak noise. Unusual behavior in a $=1.5,2,2.5$, and 3 is visible. Except in [1.5-3] regime, the dominant frequency is in the alpha band. (B): The value of dominant frequency in different coupling coefficient for ten runs. A jump phenomenon in frequency in the [1.5-3] regime is seen. 
A

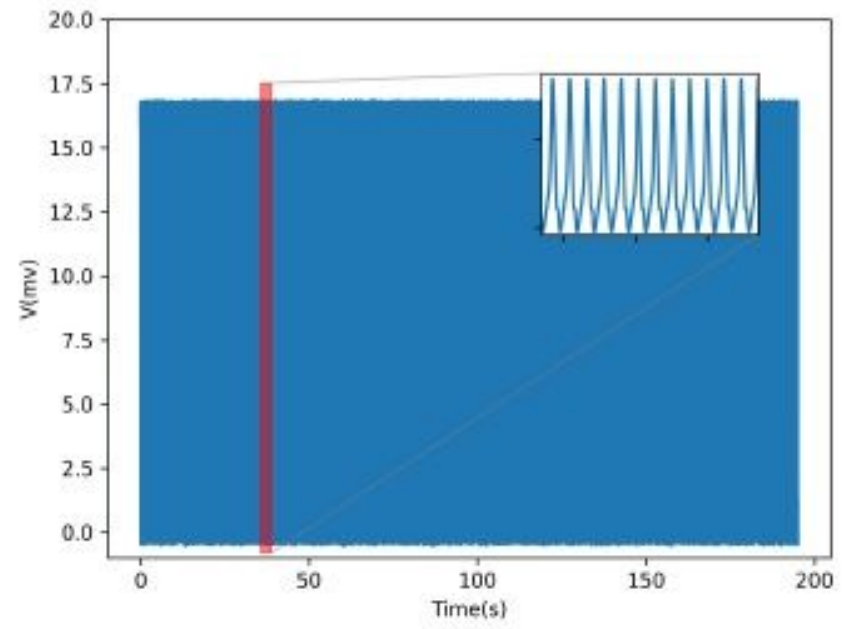

C

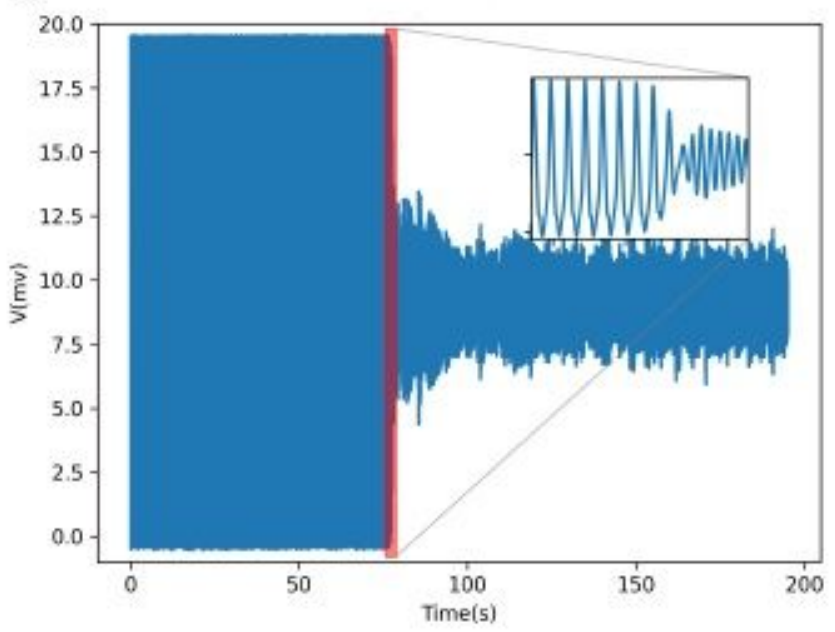

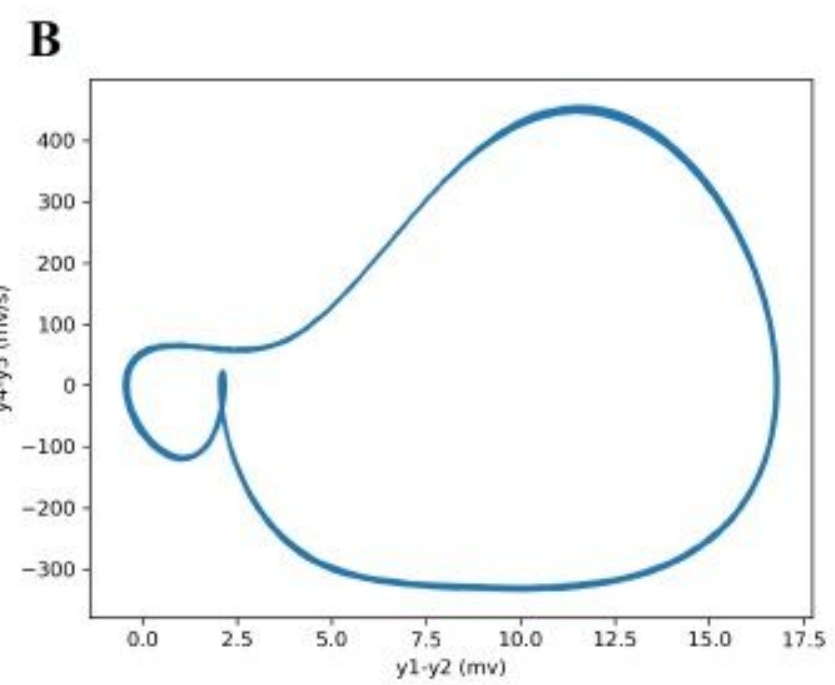

D

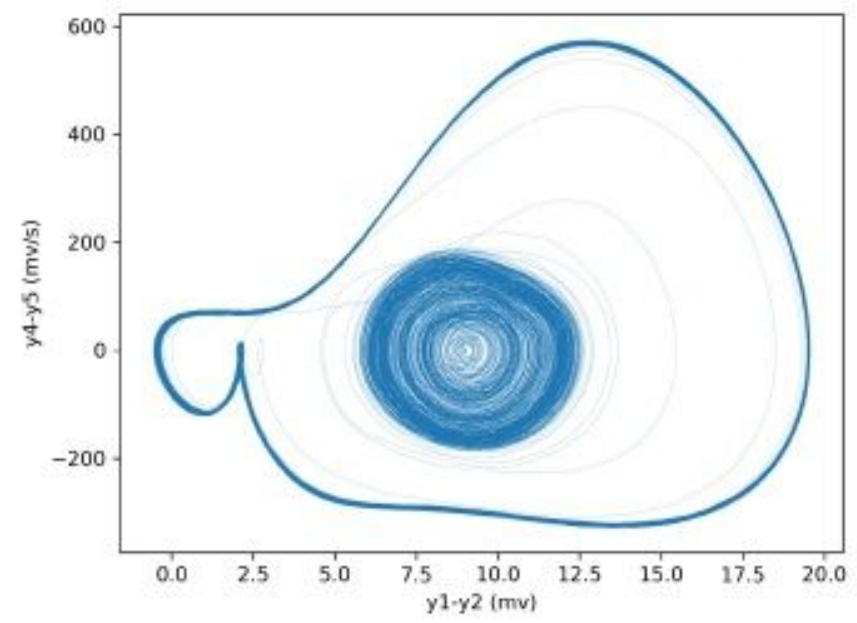

\section{Figure 6}

Two possible behaviors in [1.5-3] area ((A), (C)). High amplitude oscillations appear in this regime that either change to low amplitude oscillations (panel (C) or not (panel (A). Their phase spaces is composed of 2 limit cycles (panel (D) or just one limit cycle (panel (B) respectively. The inset of (A) and (C) shows a specific segment of signal for 3 seconds. 

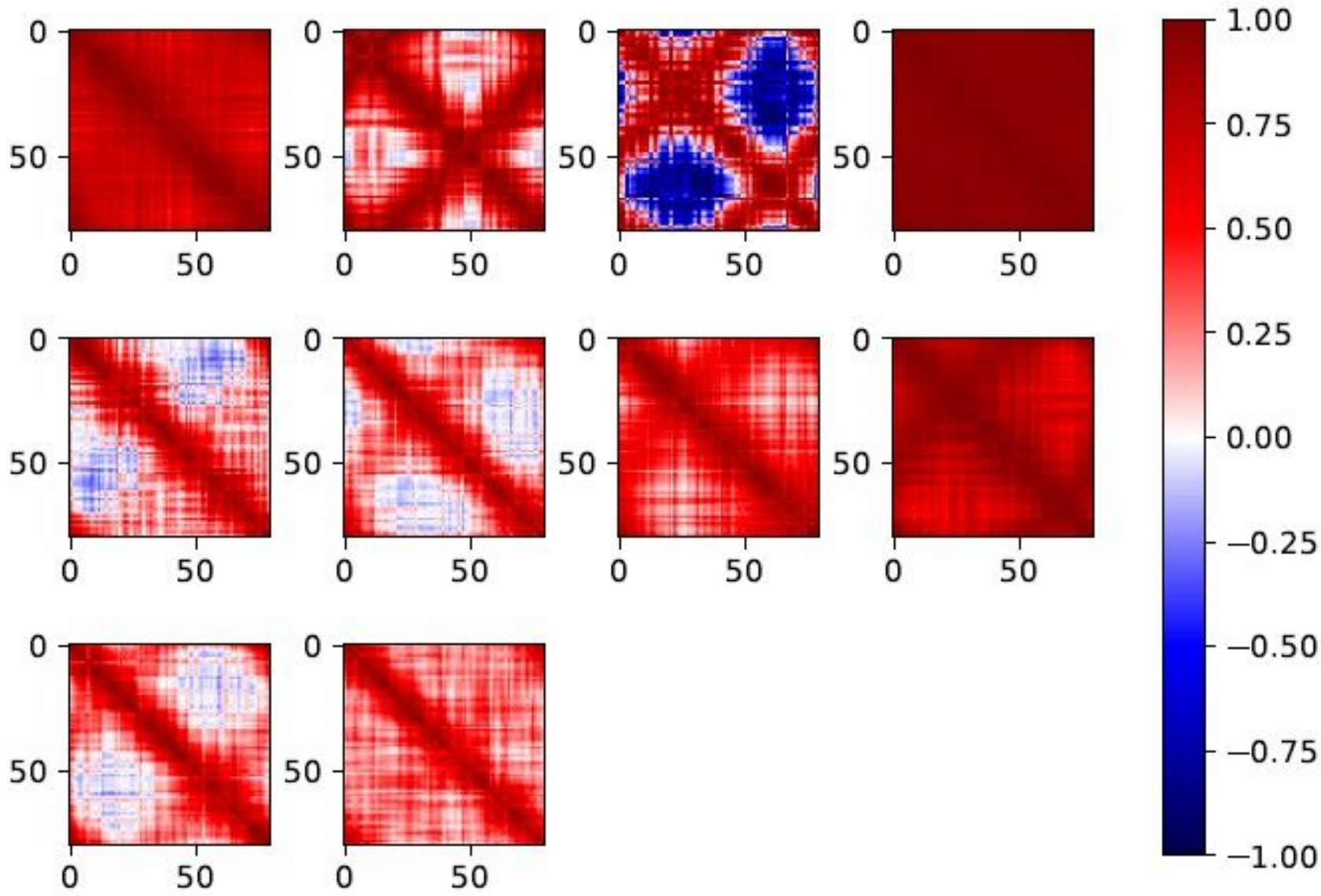

$-1.00$

Figure 7

Correlation matrices in $\mathrm{a}=3$ over 10 repetitions during weak input. Local and global synchronizations are seen. 
A

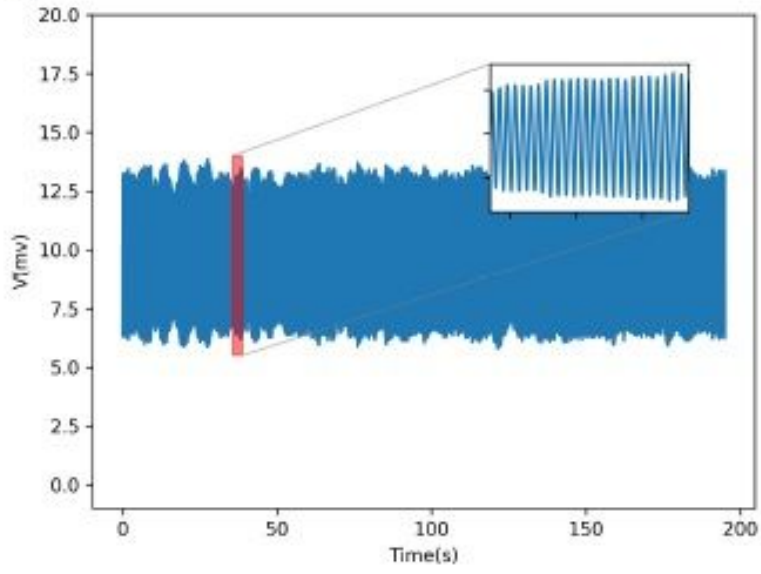

C

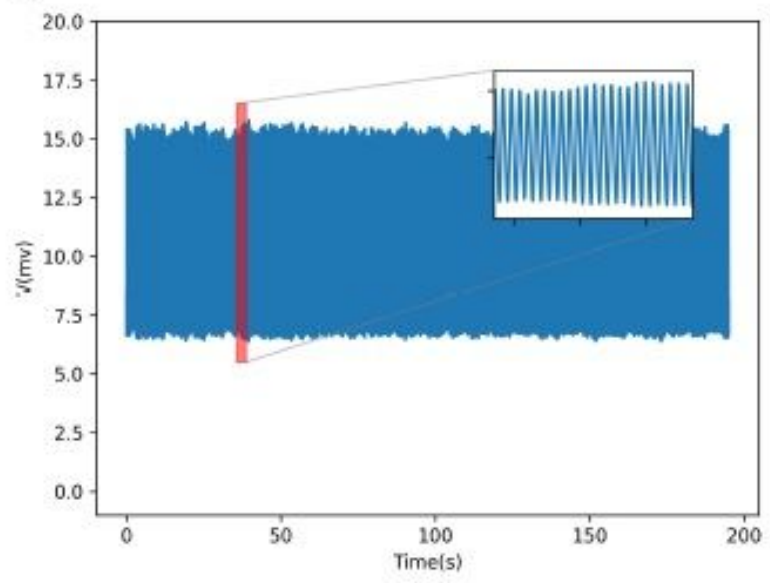

E

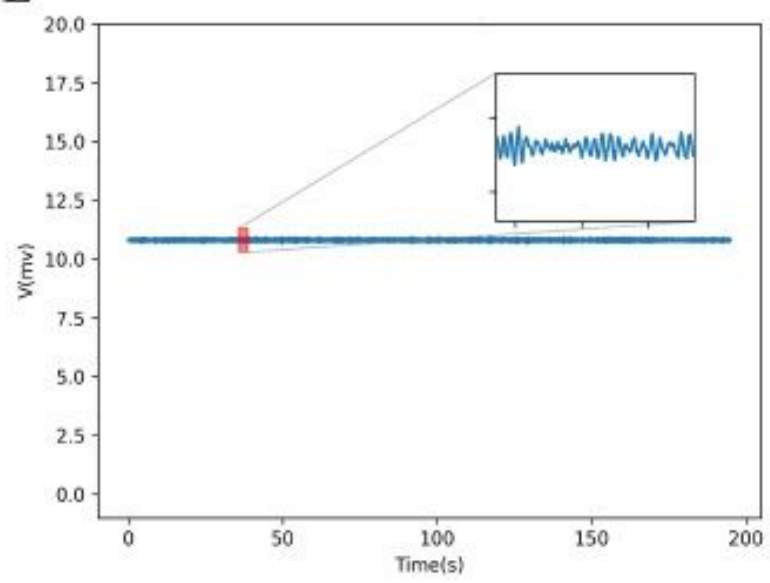

B

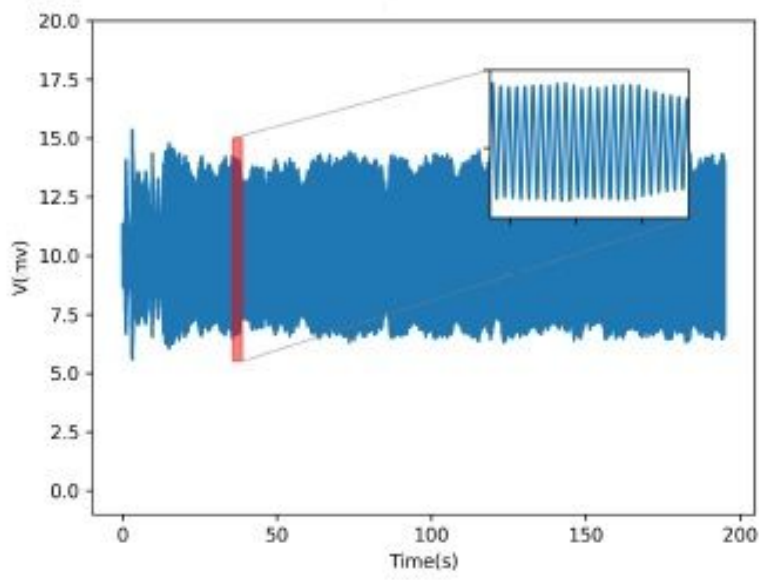

D

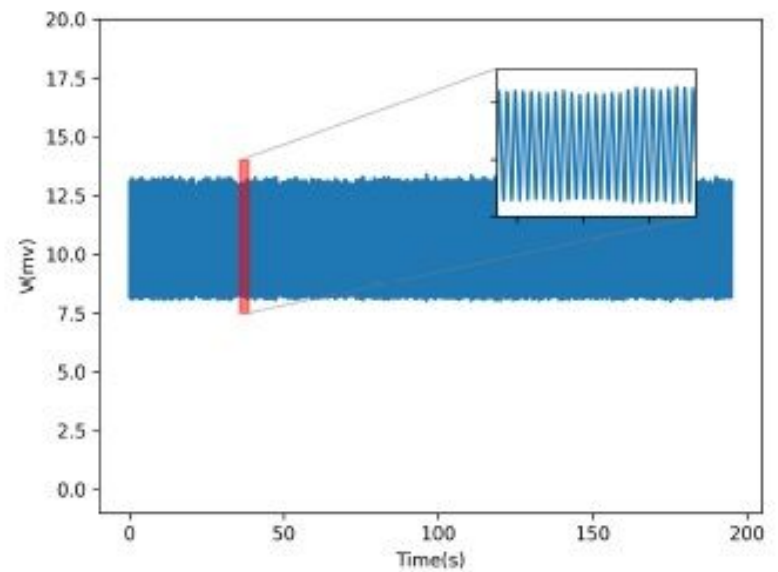

\section{Figure 8}

Time series of the mean of the network activity for $a=3.5(A), 4(B), 4.5(C), 5$ (D), and 5.5 (E). The inset of signals shows a specific segment of signal for 3 seconds. In a $=5.5$, each node leaves the limit cycle and take in resting state (fixed-point). 
A

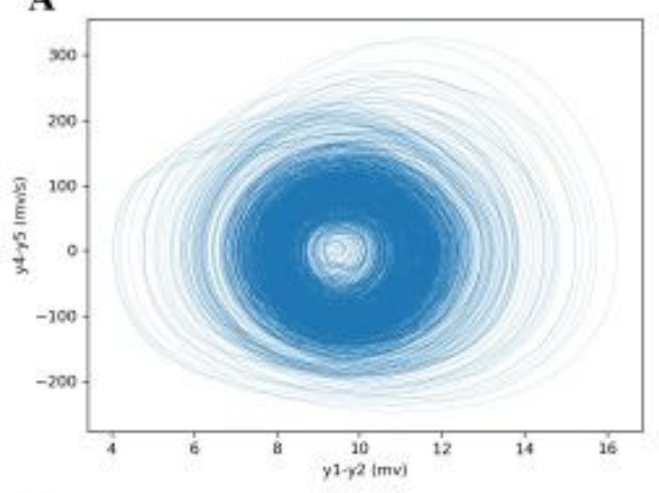

C

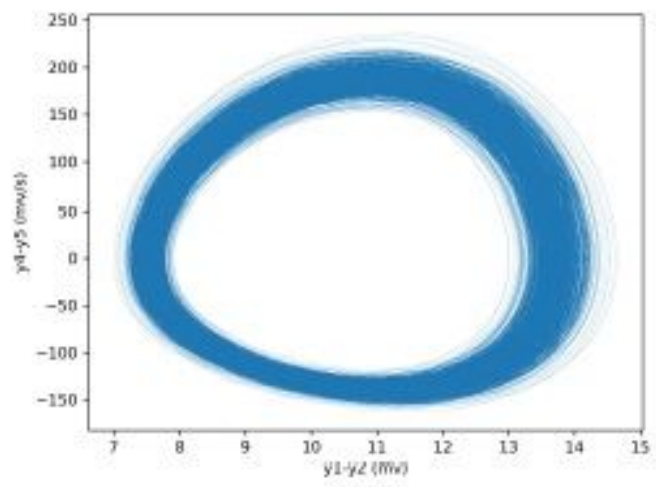

$\mathbf{E}$

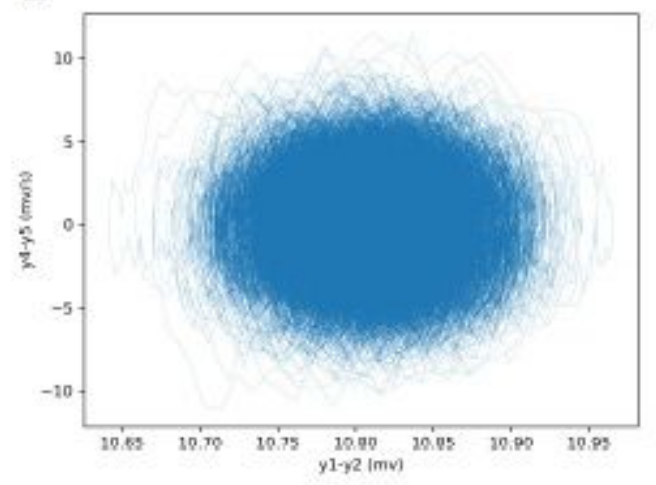

B

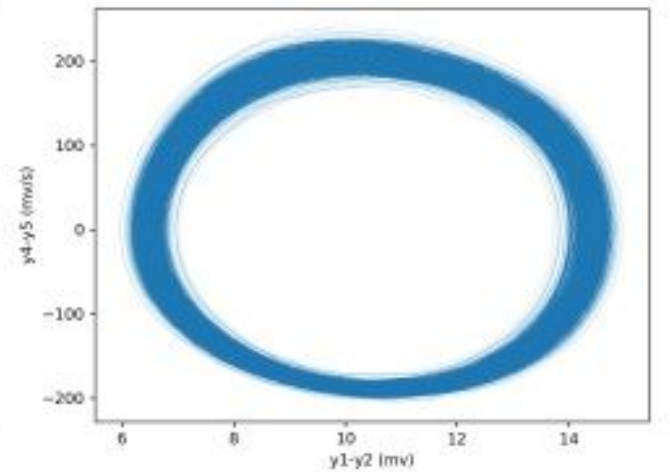

D

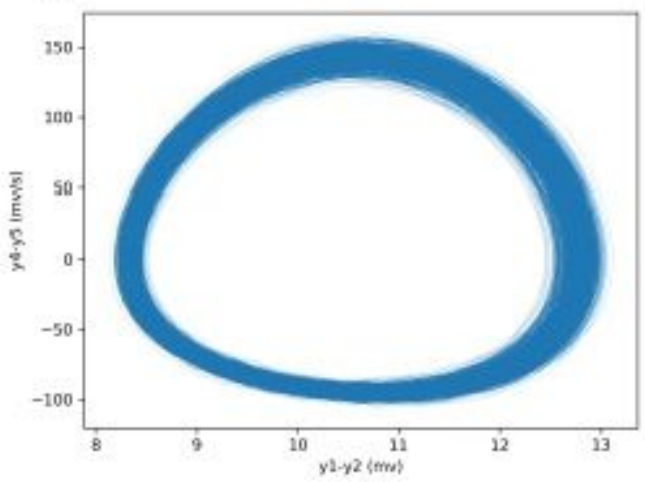

Figure 9

The phase spaces for the time series in figure 8 . In the [3.5-5], the increase of synchronization is seen. 
A

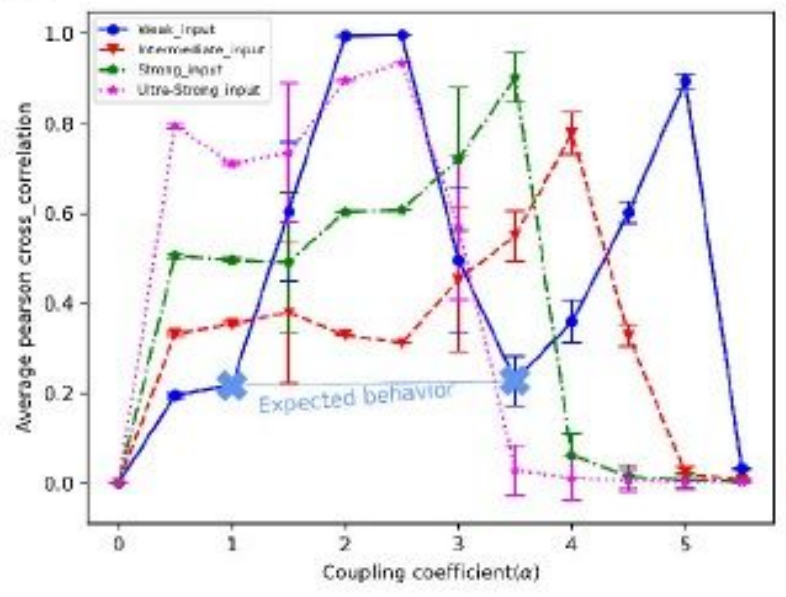

B

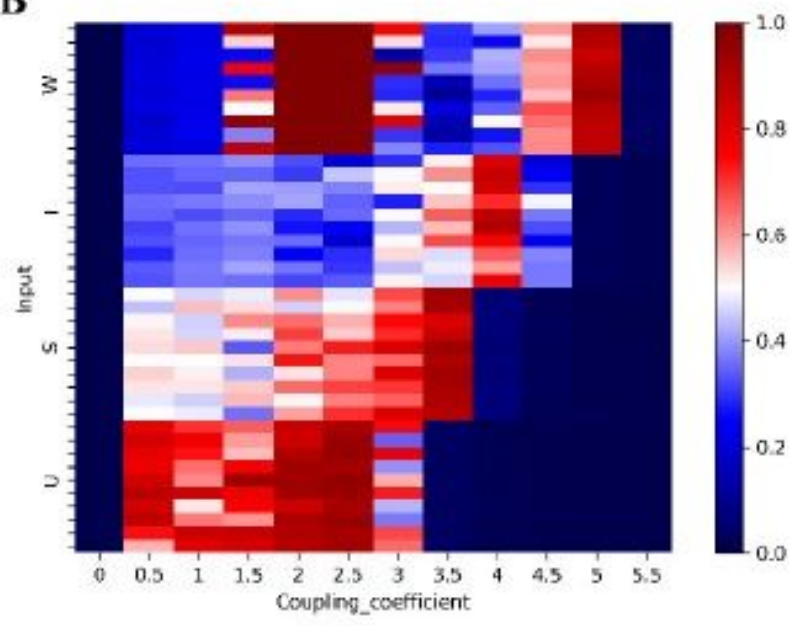

Figure 10

(A): The mean of the Pcc matrix versus coupling coefficient for four levels of input. Lower intensity input needs a larger coupling coefficient to reach its Pcc maximum. Expected behavior in the weak input level went forward as specified trend between two ' $X$ ' marks. (B): The heat-map of the mean of the Pcc matrix (W:weak, l:intermediate, S:strong, U:ultra-strong)

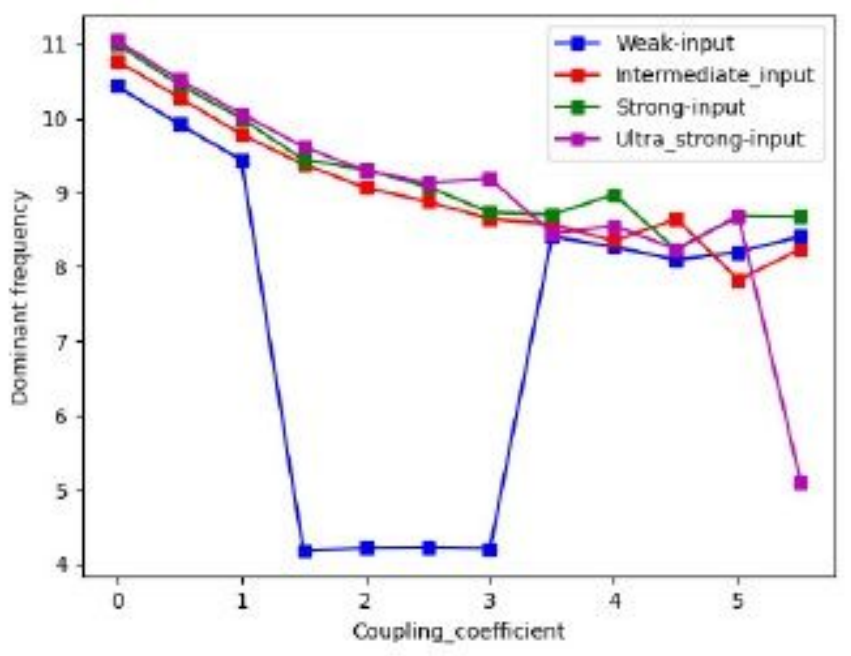

Figure 11

Dominant frequency as a function of the coupling coefficient. Increase coupling coefficient decreases the dominant frequency. 

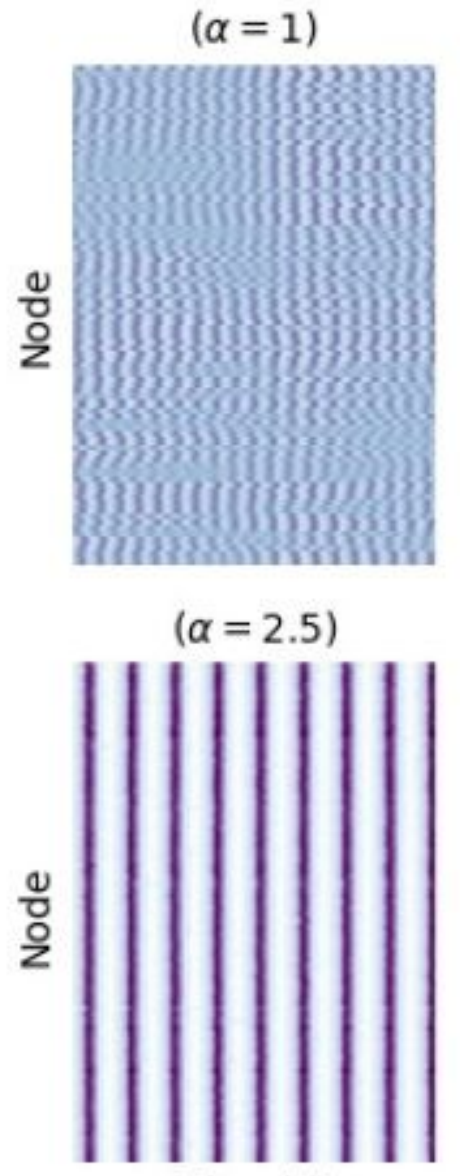

Time(s)
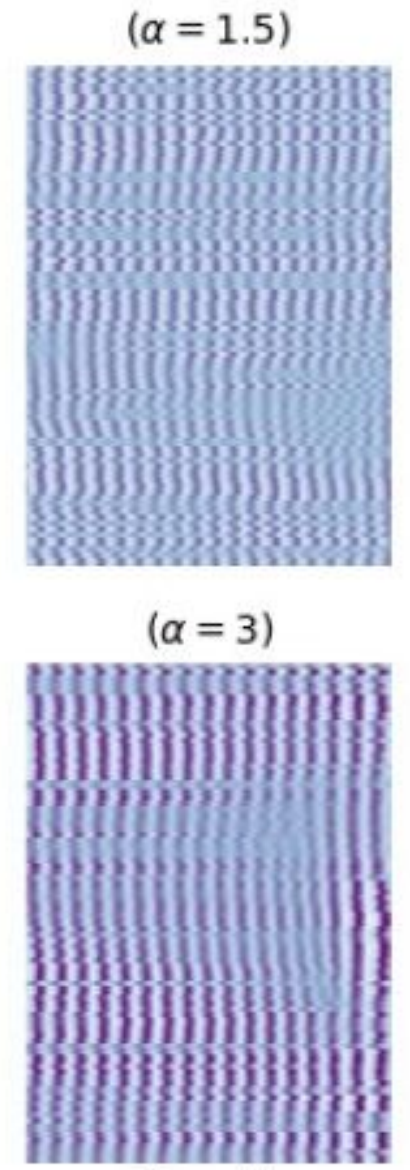

Time(s)
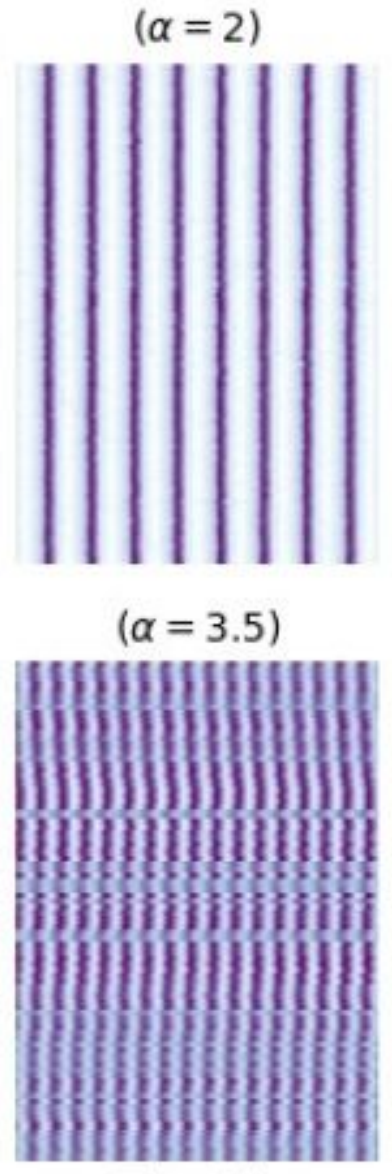

Time(s)

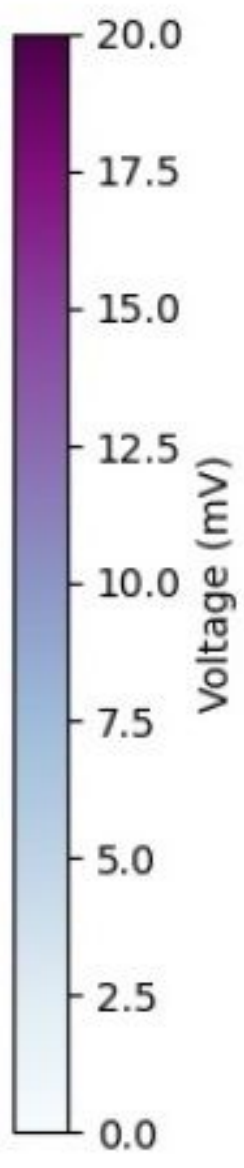

Figure 12

The spatiotemporal matrices of the mean signal for different values of coupling coefficient. In a = $1.5(3)$, there is a phase transition from disordered (ordered) to ordered (disordered) states, and the system in these coupling strengths is being on the edge of phase synchronization.

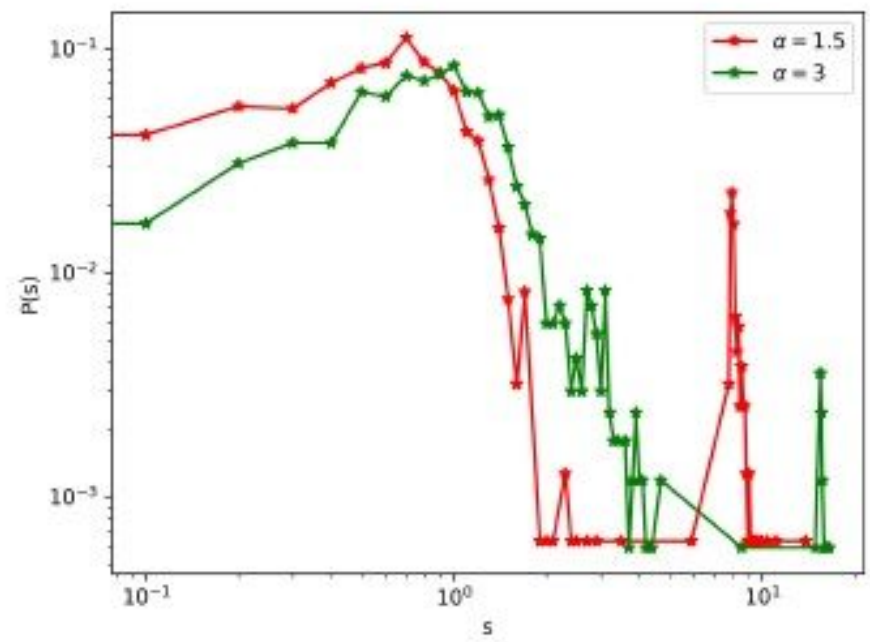


Figure 13

The distribution function of the size of avalanche in the weak input level at $a=1.5,3$. Neither of them shows power-law behavior.

A

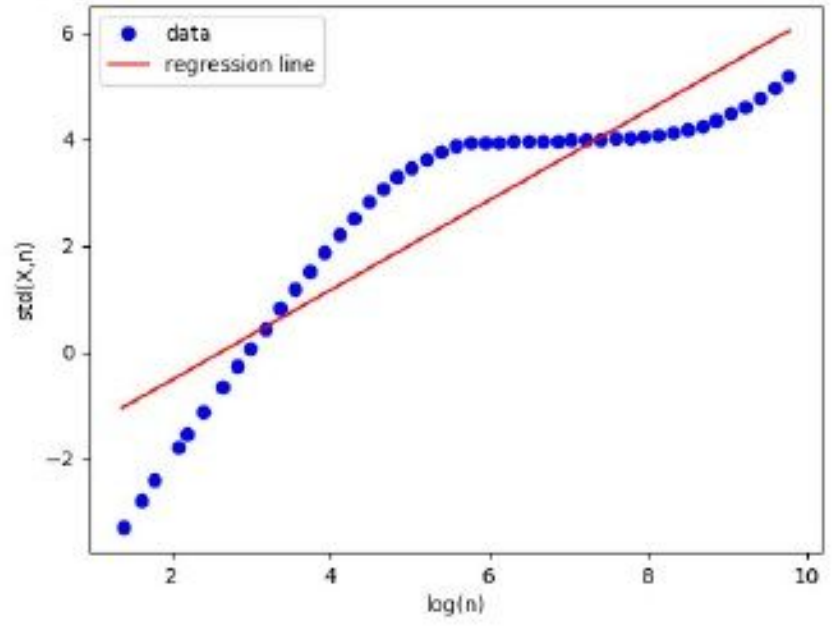

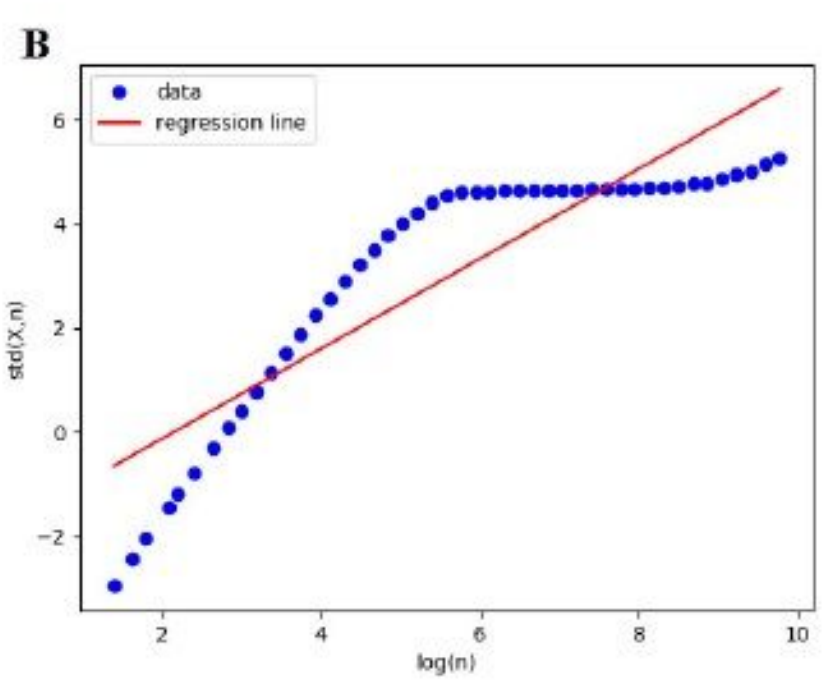

\section{Figure 14}

The fluctuation plot for $a=1.5(A)$ and $a=3(B)$ in the weak input level with the linear fit (red) in the loglog plot. This trend of data is piecewise linear and the linear fit is not suitable for them. So, LRTCs do not exist at these points. 